\title{
THE MATHEMATICS OF SECOND QUANTIZATION
}

BY

\author{
J. M. COOK \\ INTRODUCTION
}

The following is an attempt to construct a mathematical model for use in quantum field theory.

Although few nonspecialists have had opportunity to become familiar with the language of modern pure mathematics, quantum theory seems to have reached a point where it must use that language if it is to find a genuine escape from the divergence difficulties. Divergence can not be properly coped with when convergence itself has never been rigorously defined. In the classical analysis of real and complex numbers, results, even correct results, can be obtained by algebraic manipulation of formal power series; but these numbers are not just algebras, they are topological algebras, and only with Cauchy's introduction of the epsilon-delta treatment was mathematics provided an explicit method of separating sense from nonsense. Similarly, in the modern analysis of infinite-dimensional algebras results can be obtained by algebraic manipulation of formal expressions, but these results of ten require topological justification.

One standard way of introducing a topology into the algebra of observables is to make them operators on a Hilbert space. This method, which does not seem to be extensively employed in quantum electrodynamics, can be used to construct a mathematically rigorous formalism the manipulation of which is directly followable by one's physical intuition. This construction requires the exercise of two dissimilar disciplines, mathematics and physics, so the exposition is divided into two parts upon which relative emphasis can be adjusted to suit individual tastes. In particular, physicists can greatly simplify the mathematics by ignoring: (1) operator-domain considerations (as is done here in the derivation of the Yukawa-potential); (2) discussions involving the group algebra of the symmetric group (since only the FermiDirac and Bose-Einstein cases have ever actually occurred); (3) material depending on the simply-connected covering group of the Lorentz group (since it is not needed to derive Maxwell's equations). However, Part I is empty, unmotivated mathematics without Part II; and Part II does not exist without Part I. The two are designed to be read, not consecutively, but in parallel. Sections are numbered accordingly.

I would like to thank Professor I. E. Segal for liberal use of his time and advice in the preparation of this paper. It is to be submitted to the Depart-

Received by the editors December 26, 1951. 
ment of Mathematics of the University of Chicago in partial fulfillment of requirements for the Ph.D. Most of the work was done under contract with the Office of Naval Research.

\section{Part I. Mathematical preparation}

1. Definition 1. Let $\mathfrak{F}=\sum_{n=0}^{\infty} \oplus \Re^{(n)}$, where $\Re$ is a given Hilbert space of arbitrary dimension and $\Re^{(n)}=\Re \otimes \cdots \otimes \Re$ is the tensor product of $\Re$ with itself $n$ times.

(For a definition of the tensor product (direct product) see [6, Part I, chap. II].) Let $\Re^{(0)}$ be the one-dimensional Hilbert space of complex numbers; and let $\mathfrak{j}$ be the natural isomorphism $\mathfrak{R}^{(n)} \rightarrow O \oplus \cdots \oplus O \oplus \Re^{(n)} \oplus O \oplus \cdots$ of $\Re^{(n)}$ into $\mathfrak{F}$.

Definition 2. Let $A_{i}$ be densely defined, closed, linear transformations on the Hilbert spaces $\mathfrak{S}_{i}$. Then $A_{1} \otimes \cdots \otimes A_{n}$ is the densely defined, closed, linear transformation on $\mathfrak{S}_{1} \otimes \cdots \otimes \mathfrak{S}_{n}$ with domain $\mathfrak{D}_{A_{1}} \otimes \cdots \otimes D_{A_{n}}$ equal to the set of all $h$ in $\mathfrak{W}_{1} \otimes \cdots \otimes \mathfrak{W}_{n}$ such that there exists a $g$ in $\mathfrak{W}_{1} \otimes \cdots \otimes \mathfrak{W}_{n}$ with $\left(g, \phi_{1} \otimes \cdots \otimes \phi_{n}\right)=\left(h, A_{1}^{*} \phi_{1} \otimes \cdots \otimes A_{n}^{*} \phi_{n}\right)$ for all $\phi_{i}$ in $\mathfrak{D}_{A_{i}^{*}} ;$ in which case $\left(A_{1} \otimes \cdots \otimes A_{n}\right) h=g$.

$\mathfrak{D}_{A_{i}^{*}}$ is dense in $\mathfrak{T}_{i}\left[8\right.$, p. 29], so the linear set generated by $\mathfrak{D}_{A_{1}} \otimes \ldots$ $\otimes \mathfrak{D}_{A_{n}}{ }^{*}$ is dense in $\mathfrak{S}_{1} \otimes \cdots \otimes \mathfrak{W}_{n}$, and $\left(A_{1} \otimes \cdots \otimes A_{n}\right) h$ is uniquely defined when it exists. If $\phi_{i} \in \mathfrak{D}_{A_{i}}$, then $\left(A_{1} \otimes \cdots \otimes A_{n}\right) \phi_{1} \otimes \cdots \otimes \phi_{n}=A_{1} \phi_{1} \otimes \cdots$ $\otimes A_{n} \phi_{n}$, so $A_{1} \otimes \cdots \otimes A_{n}$ is densely defined. It is closed and linear by continuity and linearity of the inner product.

Now, making the conventions: (1) $[A, B]=A B-B A ;(2) A^{(n)}=A \otimes \cdots$ $\otimes A$ operates on $\Re^{(n)}$; (3) $\delta(i, j)=0$ or 1 according as $i \neq j$ or $i=j$; (4) $A^{0}=I$; (5) $\sum_{i=1}^{n} A^{\delta(i, 1)} \otimes \cdots \otimes A^{\delta(i, n)}=0$ if $n=0$; (6) $T^{\sim}$ is (when it exists) the minimal closed linear extension of the transformation $T$; we define a mapping $\Omega$ by

DEFINITION 3. If $A$ is a densely defined, closed, linear transformation on $\Re$, then $\Omega(A)$ is the densely defined, closed, linear transformation $\Omega(A)=\sum_{n=0}^{\infty}$ $\oplus\left(\sum_{i=1}^{n} A^{\delta(i, 1)} \otimes \cdots \otimes A^{\delta(i, n)}\right)^{\sim}$ on $\mathfrak{F}$.

$\mathfrak{D}_{\left(\Sigma_{i=1}^{*} A^{\delta(t, 1)} \otimes \cdots \otimes A \delta(t, n)\right)^{*}}$ contains all $\phi_{1} \otimes \cdots \otimes \phi_{n}$ such that $\phi_{i} \in \mathfrak{D}_{A^{*}}$, so $\left(\sum_{i=1}^{n} A^{\delta(i, 1)} \otimes \cdots \otimes A^{\delta(i, n)}\right)^{*}$ is densely defined and $\left(\sum_{i=1}^{n} A^{\delta(i, 1)} \otimes \cdots\right.$ $\left.\otimes A^{\delta(i, n)}\right)^{* *}=\left(\sum_{i=1}^{n} A^{\delta(i, 1)} \otimes \cdots \otimes A^{\delta(i, n)}\right)^{\sim}$ exists [8, p. 30].

Theorem 1. If $A$ is normal, then so is $\Omega(A)$, and $\Omega(A)^{*}=\Omega\left(A^{*}\right)$.

Proof. We need only prove $\left(\sum_{i=1}^{n} A^{\delta(i, 1)} \otimes \cdots \otimes A^{\delta(i, n)}\right)^{\sim}$ normal and $\left(\sum_{i=1}^{n} A^{\delta(i, 1)} \otimes \cdots \otimes A^{\delta(i, n)}\right)^{*}=\left(\sum_{i=1}^{n} A^{* \delta(i, 1)} \otimes \cdots \otimes A^{* \delta(i, n)}\right)^{\sim}[8, \mathrm{pp}$. 34 and 32 ].

Let $\left\{E_{\lambda}\right\}$ be $A$ 's resolution of the identity. Then $\left\{E_{\lambda_{1}} \otimes \cdots \otimes E_{\lambda_{n}}\right\}$ is an $n$-parameter resolution of $I^{(n)}\left[8\right.$, p. 19], and $\sum_{i=1}^{n} A^{\delta(i, 1)} \otimes \cdots \otimes A^{\delta(i, n)}$ $=\sum_{i=1}^{n} \int \lambda_{i} d E_{\lambda_{1}} \otimes \cdots \otimes E_{\lambda_{n}} \subseteq \int\left(\sum_{i=1}^{n} \lambda_{i}\right) d E_{\lambda_{1}} \otimes \cdots \otimes E_{\lambda_{n}}$ which is normal $[8$, pp. 22, 45, and 47]. Therefore 


$$
\left(\sum_{i=1}^{n} A^{\delta(i, 1)} \otimes \cdots \otimes A^{\delta(i, n)}\right)^{\sim} \subseteq \int\left(\sum_{i=1}^{n} \lambda_{i}\right) d E_{\lambda_{1}} \otimes \cdots \otimes E_{\lambda_{n}} .
$$

Now let $f \in \mathscr{D}_{\int\left(\Sigma_{\lambda_{i}}\right) d E_{\lambda_{1}} \otimes \cdots \otimes E_{\lambda_{n}}}$ and $f_{k}=\left(E_{k}-E_{-k}\right) f$ for $k=1,2, \cdots$. Then $f_{k} \in \mathfrak{D}_{\left(\Sigma_{i-1}^{n} A \delta(i, 1) \otimes \cdots \otimes A^{\delta(i, n)} \sim\right.}, \quad \lim _{k \rightarrow \infty} f_{k}=f, \lim _{k \rightarrow \infty}\left(\sum_{i=1}^{n} A^{\delta(i, 1)} \otimes \cdots\right.$ $\left.\otimes A^{\delta(i, n)}\right)^{\sim} f_{k}=\int\left(\sum_{i=1}^{n} \lambda_{i}\right) d E_{\lambda_{1}} \otimes \cdots \otimes E_{\lambda_{n}} f$, and so $f \in \mathfrak{D}_{\left(\sum_{i=1}^{n} A \delta(t, 1) \otimes \cdots \otimes \mathbb{A} \delta(, n)\right.}$, and $\left(\sum_{i=1}^{n} A^{\delta(i, 1)} \otimes \cdots \otimes A^{\delta(i, n)}\right)^{\sim}=\int\left(\sum_{i=1}^{n} \lambda_{i}\right) d E_{\lambda_{1}} \otimes \cdots \otimes E_{\lambda_{n}}$.

Similarly, $\quad\left(\sum_{i=1}^{n} A^{* \delta(i, 1)} \otimes \cdots \otimes A^{* \delta(i, n)}\right)^{\sim}=\int\left(\sum_{i=1}^{n} \lambda_{i}^{*}\right) d E_{\lambda_{1}} \otimes \cdots$ $\otimes E_{\lambda_{n}}=\left(\sum_{i=1}^{n} A^{\delta(i, 1)} \otimes \cdots \otimes A^{\delta(i, n) *}\right)^{\sim}$.

Corollary 1. The normal operators $A$ and $B$ commute if and only if $\Omega(A)$ and $\Omega(B)$ commute.

COROLlary 2. If $A$ and $B$ are densely defined, closed, linear transformations such that $A \geqq B$ and at least one is bounded, then $\Omega(A) \geqq \Omega(B)$.

Proof. Let $(A-B)^{\prime} \geqq 0$ be a self-adjoint extension of $A-B \geqq 0[8$, p. 35]. Then $\Omega\left((A-B)^{\prime}\right) \geqq 0$ also, as follows from the proof of the theorem. Since $\Omega(A)-\Omega(B)$ is densely defined, we shall be done when we have proved $\Omega\left((A-B)^{\prime}\right) \supseteq \Omega(A)-\Omega(B)$, i.e., when we have proved $(A-B)^{\prime} \otimes I \supseteq A \otimes I$ $-B \otimes I$.

Let $f \in \mathfrak{D}_{A} \otimes I-B \otimes I$. Then $\left((A \otimes I-B \otimes I) f, \phi_{1} \otimes \phi_{2}\right)=\left(f,\left(A^{*}-B^{*}\right) \phi_{1} \otimes \phi_{2}\right)$ for all $\phi_{1} \otimes \phi_{2} \in \mathfrak{D}_{A^{*}-B^{*}} \otimes \mathfrak{D}_{I}$. But either $A$ or $B$ is bounded so $(A-B)^{*}=A^{*}$ $-B^{*}\left[8\right.$, p. 29]; and $A-B \subseteq(A-B)^{\prime}$. Therefore $A^{*}-B^{*}=(A-B)^{*}$ $\supseteq\left(A-B^{*}\right)^{\prime}, \quad$ and $\left((A \otimes I-B \otimes I) f, \phi_{1} \otimes \phi_{2}\right)=\left(f, \quad\left(A-B^{*}\right)^{\prime} \phi_{1} \otimes \phi_{2}\right)$ for all $\phi_{1} \otimes \phi_{2} \in \mathfrak{D}_{\left(A-B^{*}\right)}{ }^{\prime} \otimes \mathfrak{D}_{I} . \quad$ Therefore $\quad(A \otimes I-B \otimes I) f=\left((A-B)^{\prime} \otimes I\right) f$ and $f$ $\in \mathfrak{D}_{(A-B)^{\prime} \otimes I},(A-B)^{\prime} \otimes I \supseteq A \otimes I-B \otimes I$.

THEOREM 2. If $A$ and $B$ are bounded linear transformations on $\Re$, then: (a) $\Omega(\alpha A+\beta B)=(\alpha \Omega(A)+\beta \Omega(B))^{\sim}$, (b) $\Omega([A, B])=([\Omega(A), \Omega(B)])^{\sim}$, (c) $A \geqq B$ implies $\Omega(A) \geqq \Omega(B)$, (d) $\Omega(A)^{*}=\Omega\left(A^{*}\right)$, (e) $\mathfrak{D}_{\Omega(A)}=\mathfrak{F}$ implies $A=0$.

Proof. (a) Let $f \in \mathfrak{D}_{\Omega(\alpha A+\beta B)}$, and $\left(I^{(0)} \oplus \cdots \oplus I^{(n)} \oplus O^{(n+1)} \oplus O^{(n+2)} \cdots\right) f$ $=f_{n}$. Then $\lim _{n \rightarrow \infty} f_{n}=f$ and $\lim _{n \rightarrow \infty} \Omega(\alpha A+\beta B) f_{n}=\Omega(\alpha A+\beta B) f$. But $f_{n}$ $\in \mathfrak{D}_{\alpha \Omega(A)+\beta \Omega(B)}$ and $\Omega(\alpha A+\beta B) \supseteq \alpha \Omega(A)+\beta \Omega(B)$. Therefore $f \in \mathfrak{D}_{(\alpha \Omega(A)+\beta \Omega(B))}$ and $\Omega(\alpha A+\beta B) f=(\alpha \Omega(A)+\beta \Omega(B)) f$.

(b) $[\Omega(A), \Omega(B)] \subseteq \sum_{n=0}^{\infty} \oplus\left[\sum_{i=1}^{n} A^{\delta(i, 1)} \otimes \cdots \otimes A^{\delta(i, n)}, \sum_{j=1}^{n} B^{\delta(j, 1)}\right.$ $\left.\otimes \cdots \otimes B^{\delta(j, n)}\right]=\sum_{n=0}^{\infty} \oplus \sum_{i, j=1}^{n}\left[A^{\delta(i, 1)} \otimes \cdots \otimes A^{\delta(i, n)}, B^{\delta(i, 1)} \otimes \cdots\right.$ $\left.\otimes B^{\delta(i, n)}\right]=\sum_{n=0}^{\infty} \oplus \sum_{i=1}^{n}\left[A^{\delta i, 1)} \otimes \cdots \otimes A^{\delta(i, n)}, \quad B^{\delta(i, 1)} \otimes \cdots \otimes B^{\delta(i, n)}\right]$ $=\sum_{n=0}^{\infty} \oplus \sum_{i=1}^{n}[A, B]^{\delta(i, 1)} \otimes \cdots \otimes[A, B]^{\delta(i, n)}=\Omega([A, B])$. Now proceed as in (a).

(c) Follows from Corollary 2.

(d) Obvious.

(e) If $A \neq 0$, then there exists an element $\phi \in \Re$ such that $\|\phi\|=1, A \phi \neq 0$. Then $\sum_{n=1}^{\infty} \oplus(1 / n) \phi \otimes \cdots \otimes \phi \in \mathfrak{F}$, but $\left\|\Omega(A) \sum_{n=1}^{\infty} \oplus(1 / n) \phi \otimes \cdots \otimes \phi\right\|^{2}$ 
$=\sum_{n=1}^{\infty} 1 / n^{2} \sum_{i, j=1}^{n}\left(A^{\delta(i, 1)} \phi \otimes \cdots \otimes A^{\delta(i, n)} \phi, \quad A^{\delta(j, 1)} \phi \otimes \cdots \otimes A^{\delta(j, n)}\right)$ $=\left(\sum_{n=1}^{\infty}\left(1 / n^{2}\right)\left(n\|A \phi\|^{2}+n(n-1)|(A \phi, \phi)|^{2}\right) \geqq\|A \phi\|^{2} \sum_{n=1}^{\infty} 1 / n=\infty\right.$.

Theorem 3. If $H$ and $A$ are linear transformations on $\Re$ such that $H$ is self-adjoint and $A$ is densely defined and closed, then

$$
\exp (i \Omega(H)) \Omega(A) \exp (-i \Omega(H))=\Omega(\exp (i H) A \exp (-i H)) .
$$

Proof. $\exp (i \Omega(H))=\sum_{n=0}^{\infty} \oplus \exp \left(i\left(\sum_{j=1}^{n} H^{\delta(j, 1)} \otimes \cdots \otimes H^{\delta(j, n)}\right)^{\sim}\right)$ $=\sum_{n=0}^{\infty} \oplus \prod_{j=1}^{n} \exp \left(i H^{\delta(j, 1)} \otimes \cdots \otimes H^{\delta(j, n)}\right)=\sum_{n=0}^{\infty} \oplus \prod_{j=1}^{n} \exp (i H)^{\delta(j, 1)}$ $\otimes \cdots \otimes \exp (i H)^{\delta(j, n)}$, so

$$
\exp (i \Omega(H))=\sum_{n=0}^{\infty} \oplus \exp (i H)^{(n)} .
$$

We are dealing with automorphisms of $\Re, \Re^{(n)}$, and $\mathfrak{F}$, so $\exp (i \Omega(H)) \Omega(A) \exp (-i \Omega(H))=\sum_{n=0}^{\infty} \oplus \exp (i H)^{(n)}\left(\sum_{j=1}^{n} A^{\delta(j, 1)} \otimes \cdots\right.$ $\left.\otimes A^{\delta(j, n)}\right)^{\sim} \exp (-i H)^{(n)}=\sum_{n=0}^{\infty} \oplus\left(\sum_{j=1}^{n} \exp (i H)^{(n)} A^{\delta(j, 1)} \otimes \cdots \otimes A^{\delta(j, n)}\right.$ $\left.\exp (-i H)^{(n)}\right)^{\sim}=\sum_{n=0}^{\infty} \oplus\left(\sum_{j=1}^{n} \exp (i H) A^{\delta(j, 1)} \quad \exp \quad(-i H) \otimes \cdots\right.$ $\left.\otimes \exp (i H) A^{\delta(j, n)} \exp (-i H)\right)^{\sim}=\sum_{n=0}^{\infty} \oplus\left(\sum_{j=1}^{n}(\exp (i H) A \exp (-i H))^{\delta(j, 1)}\right.$ $\left.\otimes \cdots \otimes(\exp (i H) A \exp (-i H))^{\delta(i, n)}\right)^{\sim}=\Omega(\exp (i H) A \exp (-i H))$.

2. To every permutation $\pi$, in the symmetric group $\Pi_{n}$ of degree $n$, there corresponds a unitary operator $U_{\pi}$ on $\Re^{(n)}$ uniquely defined as the bounded linear extension of the operation $U_{\pi} \psi_{1} \otimes \cdots \otimes \psi_{n}=\psi_{\pi(1)} \otimes \cdots \otimes \psi_{\pi(n)}$ on decomposable tensors. The $n$ !-dimensional ring $G_{n}$ generated by $\left\{U_{\pi}\right\}$ is isomorphic with the group algebra $[9, \S 14]$ of $\Pi_{n}$.

To every $\phi$ in $\Re$ there corresponds the linear transformation $(\phi \otimes)$ on $\mathfrak{F}$ uniquely defined as the bounded linear extension of the operation $(\phi \otimes) \dot{i} \psi_{1}$ $\otimes \cdots \otimes \psi_{n}=i \phi \otimes \psi_{1} \otimes \cdots \otimes \psi_{n}$. Then $\|(\phi \otimes)\|=\|\phi\|$, and $(\phi \otimes)^{*}$ is the bounded linear extension of $(\phi \otimes) * i \psi_{1} \otimes \cdots \otimes \psi_{n}=\mathrm{i}\left(\psi_{1}, \phi\right) \psi_{2} \otimes \cdots \otimes \psi_{n}$ (where $(\phi \otimes)^{*} \alpha=0$ for $\alpha \in \Re^{(0)}$ ).

Let $G$ be a function which assigns to every $n=0,1,2, \cdots$ an operator $G_{n}$ in $G_{n}$. Then

Definition 4. $\omega_{G}$ and $\omega_{G}{ }^{*}$ map $\Re$ into the set of all densely defined, closed, linear transformations on $\mathfrak{F}$ by $\omega_{G}(\phi)=\left(\sum_{n=0}^{\infty} \oplus G_{n}\right)(\phi \otimes), \quad \omega_{G}^{*}(\phi)$ $=\left((\phi \otimes) *\left(\sum_{n=0}^{\infty} \oplus G_{n}^{*}\right)\right)^{\sim}$.

$\omega_{G}(\phi)$ is closed because $\left(\sum_{n=0}^{\infty} \oplus G_{n}\right)$ is closed and $(\phi \otimes)$ is bounded. If $f \in \mathfrak{D}_{(\phi \otimes)^{*}\left(\Sigma_{n-0}^{\infty} \otimes G_{n}^{*}\right)}$ and $g \in \mathfrak{D}_{\omega_{G}(\phi)}$, then $\left((\phi \otimes)^{*}\left(\sum_{n=0}^{\infty} \oplus G_{n}{ }^{*}\right) f, g\right)$ $=\left(f,\left(\sum_{n=0}^{\infty} \oplus G_{n}\right)(\phi \otimes) g\right)$, so $\omega_{G}(\phi) \subseteq\left((\phi \otimes) *\left(\sum_{n=0}^{\infty} \oplus G_{n}{ }^{*}\right)\right)^{*}$ is densely defined and $\omega_{\mathrm{G}}{ }^{*}(\phi)=\left((\phi \otimes)^{*}\left(\sum_{n=0}^{\infty} \oplus G_{n}^{*}\right)\right)^{\sim}=\left((\phi \otimes)^{*}\left(\sum_{n=0}^{\infty} \oplus G_{n}^{*}\right)\right)^{* *}$ exists [8, p. 30], and equals $\left(\left(\sum_{n=0}^{\infty} \oplus G_{n}^{*}\right)^{*}(\phi \otimes)^{* *}\right)^{*}$ by [8, p. 29]. Therefore

$$
\omega_{G}(\phi)^{*}=\omega_{G}^{*}(\phi), \quad \omega_{G}^{*}(\phi)^{*}=\omega_{G}(\phi) .
$$

$\alpha_{1} \omega_{G}\left(\phi_{1}\right)+\alpha_{2} \omega_{G}\left(\phi_{2}\right)$ and $\alpha_{1}^{*} \omega_{G}{ }^{*}\left(\phi_{1}\right)+\alpha_{2}^{*} \omega_{G}{ }^{*}\left(\phi_{2}\right)$ are densely defined linear transformations such that $\left(\alpha_{1} \omega_{G}\left(\phi_{1}\right)+\alpha_{2} \omega_{G}\left(\phi_{2}\right)\right)^{\sim}=\omega_{G}\left(\alpha_{1} \phi_{1}+\alpha_{2} \phi_{2}\right)$ and 
$\left(\alpha_{1}^{*} \omega_{G}^{*}\left(\phi_{1}\right)+\alpha_{2}^{*} \omega_{G}^{*}\left(\phi_{2}\right)\right)^{\sim}=\omega_{G}^{*}\left(\alpha_{1} \phi_{1}+\alpha_{2} \phi_{2}\right)$.

Theorem 4. If $A$ is self-adjoint, then $\exp (-i \Omega(A)) \omega_{G}(\phi) \exp (i \Omega(A))$ $=\omega_{G}(\exp (-i A) \phi)$ and $\exp (-i \Omega(A)) \omega_{G}{ }^{*}(\phi) \exp (i \Omega(A))=\omega_{G}{ }^{*}(\exp (-i A) \phi)$.

Proof.

$\exp (-i \Omega(A)) \omega_{G}(\phi) \exp (i \Omega(A))$

$$
\begin{aligned}
& =\left(\sum_{n=0}^{\infty} \oplus \exp (-i A)^{(n)}\right) \omega_{G}(\phi)\left(\sum_{n=0}^{\infty} \oplus \exp (i A)^{(n)}\right) \\
& =\left(\sum_{n=0}^{\infty} \oplus G_{n}\right)\left(\sum_{n=0}^{\infty} \oplus \exp (-i A)^{(n)}\right)(\phi \otimes)\left(\sum_{n=0}^{\infty} \oplus \exp (i A)^{(n)}\right) \\
& =\left(\sum_{n=0}^{\infty} \oplus G_{n}\right)(\exp (-i A) \phi \otimes) \\
& =\omega_{G}(\exp (-i A) \phi)
\end{aligned}
$$

by (7). The second expression follows by taking adjoints.

ThEOREM 5. If $A$ is a densely defined, closed, linear transformation on $\Re$, and $\phi \in \mathfrak{D}_{A}, \psi \in \mathfrak{D}_{A^{*}}$, then $\left[\Omega(A), \omega_{G}(\phi)\right]$ and $\left[\Omega(A), \omega_{G}^{*}(\psi)\right]$ are densely defined, linear transformations on $\mathfrak{F}$ such that $\left(\left[\Omega(A), \omega_{G}(\phi)\right]\right)^{\sim}=\omega_{G}(A \phi)$ and $\left(\left[\Omega(A), \omega_{G}^{*}(\psi)\right]\right)^{\sim}=-\omega_{G}^{*}\left(A^{*} \psi\right)$.

Proof. Let $f_{n} \in \mathfrak{i} \Re^{(n)} \cap \mathfrak{D}_{\left[\Omega(A), \omega_{G}(\phi)\right]}, \psi_{i} \in \mathfrak{D}_{A^{*}}$. Then $\left[\Omega(A), \omega_{G}(\phi)\right] f_{n}$ $\in \mathrm{i}^{(n+1)}$, and $\left(\left[\Omega(A), \omega_{G}(\phi)\right] f_{n}, \dot{\mathrm{i}} \psi_{1} \otimes \cdots \otimes \psi_{n+1}\right)=\left(f_{n},\left[\omega_{G}^{*}(\phi), \Omega\left(A^{*}\right)\right] \mathrm{i} \psi_{1}\right.$ $\left.\otimes \cdots \otimes \psi_{n+1}\right)=\left(f_{n}, \quad\left[(\phi \otimes)^{*}, \quad \Omega\left(A^{*}\right)\right]\left(\sum_{n=0}^{\infty} \oplus G_{n}^{*}\right) \dot{\mathrm{j}} \psi_{1} \otimes \cdots \otimes \psi_{n+1}\right)$ $=\left(f_{n},(A \phi \otimes)^{*}\left(\sum_{n=0}^{\infty} \oplus G_{n}^{*}\right) \dot{\mathrm{i}} \psi_{1} \otimes \cdots \otimes \psi_{n+1}\right)=\left(f_{n}, \omega_{G}^{*}(A \phi) \dot{\mathrm{i}} \psi_{1} \otimes \cdots \otimes \psi_{n+1}\right)$ $=\left(\omega_{G}(A \phi) f_{n}, \mathrm{i} \psi_{1} \otimes \cdots \otimes \psi_{n+1}\right)$, so $\left[\Omega(A), \omega_{G}(\phi)\right] f_{n}=\omega_{G}(A \phi) f_{n}$.

If $f \in \mathfrak{D}_{\left[\Omega(A), \omega_{G}(\phi)\right]}$ then $f=\sum_{n=0}^{\infty} f_{n}$ with $f_{n} \in i \Re^{(n)} \cap \mathfrak{D}_{\left[\Omega(A), \omega_{G}(\phi)\right]}$, and $\left[\Omega(A), \omega_{G}(\phi)\right] f=\sum_{n=0}^{\infty}\left[\Omega(A), \omega_{G}(\phi)\right] f_{n}=\sum_{n=0}^{\infty} \omega_{G}(A \phi) f_{n}=\omega_{G}(A \phi) f$ and so $\left[\Omega(A), \omega_{G}(\phi)\right] \subseteq \omega_{G}(A \phi)$.

Since $\left[\Omega(A), \omega_{G}(\phi)\right]$ has a closed linear extension $\omega_{G}(A \phi)$, it has a closure $\left(\left[\Omega(A), \omega_{G}(\phi)\right]\right)^{\sim} \subseteq \omega_{G}(A \phi)$. Now let $f \in \mathcal{D}_{\omega_{G}(A \phi)}, f=\sum_{n=0}^{\infty} f_{n}, f_{n} \in \mathfrak{i} \Re^{(n)}$. $\left[\Omega(A), \omega_{G}(\phi)\right]$ is densely defined on $i \Re^{(n)}$, and there exists $g_{i, n} \in \mathrm{i} \Re^{(n)}$ $\cap \mathfrak{D}_{\left[\Omega(A), \omega_{G}(\phi)\right]}$ such that $\lim _{i \rightarrow \infty} g_{i, n}=f_{n} . \omega_{G}(A \phi)$ is bounded on each $i \Re^{(n)}$, so $\lim _{1 \rightarrow \infty} \omega_{G}(A \phi) g_{i, n}=\omega_{G}(A \phi) f_{n}, \quad$ i.e., $\quad \lim _{i \rightarrow \infty}\left(\left[\Omega(A), \omega_{G}(A \phi)\right]\right)^{\sim} g_{i, n}$ $=\omega_{G}(A \phi) f_{n}$. But $\left(\left[\Omega(A), \omega_{G}(\phi)\right]\right)^{\sim}$ is closed, so $f_{n} \in \mathfrak{D}_{\left(\left[\Omega(A), \omega_{G}(\phi)\right]\right)^{\sim}}$ and $\left(\left[\Omega(A), \omega_{G}(\phi)\right]\right)^{\sim} f_{n}=\omega_{G}(A \phi) f_{n}$. Now $\lim _{m \rightarrow \infty} \sum_{n=0}^{m} f_{n}=f$ and $\lim _{m \rightarrow \infty}$ $\left(\left[\Omega(A), \quad \omega_{G}(\phi)\right]\right)^{\sim} \sum_{n=0}^{m} \quad f_{n}=\lim _{m \rightarrow \infty} \quad \sum_{n=0}^{m} \quad \omega_{G}(A \phi) f_{n}=\omega_{G}(A \phi) f, \quad$ so $\stackrel{f}{f}$ $\in \mathfrak{D}_{\left(\left[\Omega(A), \omega_{G}(\phi)\right]\right)}$ and $\left(\left[\Omega(A), \omega_{G}(\phi)\right]\right)^{\sim} f=\omega_{G}(A \phi) f$. Therefore $\left(\left[\Omega(A), \omega_{G}(\phi)\right]\right)^{\sim}$ $=\omega_{G}(A \phi)$.

To prove the second expression, let $f_{n} \in \mathrm{i} \Re^{(n)} \cap \mathfrak{D}_{\left[\Omega(A), \omega_{G^{*}}(\psi)\right]}, \psi_{i} \in \mathfrak{D}_{A^{*}}$. Then 
$\left[\Omega(A), \omega_{G}^{*}(\psi)\right] f_{n} \in \mathfrak{i}^{(n-1)}$, and $\left(\left[\Omega(A), \omega_{G}^{*}(\psi)\right] f_{n}, \quad \dot{\mathrm{i}} \psi_{1} \otimes \cdots \otimes \psi_{n-1}\right)=\left(f_{n}\right.$, $\left.\left[\omega_{G}(\psi), \Omega\left(A^{*}\right)\right] \dot{i} \psi_{1} \otimes \cdots \otimes \psi_{n-1}\right)=\left(f_{n},\left(\sum_{n=0}^{\infty} \oplus G_{n}\right)\left[(\psi \otimes), \Omega\left(A^{*}\right)\right] \dot{i} \psi_{1} \otimes \cdots\right.$ $\left.\otimes \psi_{n-1}\right)=\left(f_{n}, \quad\left(\sum_{n=0}^{\infty} \oplus G_{n}\right)\left(-A^{*} \psi \otimes\right) \dot{\mathrm{i}} \psi_{1} \otimes \cdots \otimes \psi_{n-1}\right)=\left(f_{n}, \quad-\omega_{G}\left(A^{*} \psi\right) \mathrm{i} \psi_{1}\right.$ $\left.\otimes \cdots \otimes \psi_{n-1}\right)=\left(-\omega_{G}^{*}\left(A^{*} \psi\right) f_{n}, \mathrm{i} \psi_{1} \otimes \cdots \otimes \psi_{n-1}\right)$, and as before [ $\Omega(A)$, $\left.\omega_{G}^{*}(\psi)\right] f_{n}=-\omega_{G}{ }^{*}\left(A^{*} \psi\right) f_{n}$. The remainder of the proof is identical with that of the preceding case.

3. From the general theory of group algebras $[9, \S \S 14,15]$ we know that the center of $G_{n}$ is generated by a set of orthogonal projections $\left\{P_{\tau}\right\}$ indexed by the characters $\tau$ of $\Pi_{n}$, such that $P_{\tau}=(1 / n !) \sum_{\pi \in \Pi_{n}} \tau(\pi) U_{\pi}$. The corresponding resolution of the identity $I^{(n)}=\sum_{\tau} P_{\tau}$ of $\Re^{(n)}$ gives the central decomposition of $G_{n}$ into factors $P_{\tau} G_{n}$ of type $I_{m}$, where $m=m(\tau)$ is the dimensionality of that irreducible representation of $\Pi_{n}$ belonging to $\tau$. Each $P_{\tau}$ can be further decomposed into orthogonal minimal projections $P_{\tau}$ $=\sum_{i=1}^{m} P_{\tau, i}$ (by means of Young symmetrizers). The only $\tau$ such that $m(\tau)=1$, i.e., such that $P_{\tau}$ is itself minimal, are the alternating character $a_{n}\left(a_{n}(\pi)=1\right.$ or -1 according as $\pi$ is even or odd) and the identity character $s_{n}\left(s_{n}(\pi)=1\right.$ for all $\pi$ ). These two characters are the only ones which are homomorphisms of $\Pi_{n}$, and are the only ones which we shall investigate further. $P_{\alpha_{n}}$ is the projection of $\Re^{(n)}$ on its subspace of anti-symmetric tensors, $P_{s_{n}}$ is the projection of $\Re^{(n)}$ on its subspace of symmetric tensors.

To treat the anti-symmetric case first, we restrict all our operators to the subspace $\mathfrak{A}=\left(\sum_{n=0}^{\infty} P_{\alpha_{n}}\right) \mathfrak{F}$ and define $\omega_{G}$ and $\omega_{G}{ }^{*}$ by picking $G$ to be that particular function $A$ which assigns to every non-negative integer $n$ the operator $n^{1 / 2} P_{\alpha_{n}}$ in $G_{n}$. Thus $\omega_{A}(\phi)=\left(\sum_{n=0}^{\infty} \oplus n^{1 / 2} P_{\alpha_{n}}\right)(\phi \otimes)$, and $\omega_{A}{ }^{*}(\phi)$ $=\left((\phi \otimes) *\left(\sum_{n=0}^{\infty} \oplus n^{1 / 2} P_{\alpha_{n}}\right)\right)^{1 / 2}$ considered as linear transformations of $\mathfrak{A}$. $\mathfrak{A}$ reduces $\Omega(A)$, so the discussions in $\S \S 1$ and 2 apply unchanged.

For any orthonormal basis $\left\{\phi_{i}\right\}$ of $\Re$, we define an orthonormal basis $\left\{\left(\phi_{1}^{n_{1}} \phi_{2}^{n_{2}} \cdots\right)_{A}\right\}$ of $\mathfrak{A}$, where $n_{i}=0$ or 1 and $\sum_{i} n_{i}<\infty$, by $\left(\phi_{1}^{n_{1}} \phi_{2}^{n_{2}} \cdots\right)_{A}$ $=\mathrm{i}\left(\left(\sum_{i} n_{i}\right) !\right)^{1 / 2} P_{\alpha \Sigma n_{i}} \phi_{1} \otimes \cdots \otimes \phi_{1} \otimes \phi_{2} \otimes \cdots \otimes \phi_{2} \otimes \phi_{3} \otimes \cdots$. (The element $\phi_{i}$ appears $n_{i}$ times in the tensor product.) Extend the definition of $\left(\phi_{1}^{n_{1}} \phi_{2}^{n_{2}} \cdots\right)_{A}$ so that it is zero if $n_{k}>1$, or $n_{k}<0$ for any $k$.

By direct computation it is seen that

$$
\begin{aligned}
& \omega_{A}\left(\phi_{k}\right)\left(\phi_{1}^{n_{1}} \cdots \phi_{k}^{n_{k}} \cdots\right)_{A}=(-1)^{\sum_{i=1}^{k-1} n_{k}}\left(\phi_{1}^{n_{1}} \cdots \phi_{k}^{{ }^{n k+1}} \cdots\right)_{A} \text {, } \\
& \omega_{A}^{*}\left(\phi_{k}\right)\left(\phi_{1}^{n_{1}} \cdots \phi_{k}^{n_{k}} \cdots\right)_{A}=(-1)^{\sum_{i=1}^{k-1} n_{i}}\left(\phi_{1}^{n_{1}} \cdots \phi_{k}^{n_{k-1}} \cdots\right)_{A} \text {. }
\end{aligned}
$$

Therefore $\omega_{A}(\phi)$ is a bounded linear transformation on $\mathfrak{A}$ such that $\left\|\omega_{\Delta}(\phi)\right\|=\|\phi\|$ and $\omega_{A}(\alpha \phi+\beta \psi)=\alpha \omega_{A}(\phi)+\beta \omega_{A}(\psi)$.

DEFINITION 5. If $\psi_{1}, \psi_{2} \in \Re$, then $\psi_{1} \psi_{2}{ }^{*}$ is the bounded linear operator on $\Re$ defined by $\left(\psi_{1} \psi_{2}^{*}\right) \phi=\left(\phi, \psi_{2}\right) \psi_{1}$.

LeMma 1. $\omega_{A}(\phi) \omega_{A}^{*}(\psi)=\Omega\left(\phi \psi^{*}\right), \omega_{A}^{*}(\psi) \omega_{A}(\phi)=(\phi, \psi) I-\Omega\left(\phi \psi^{*}\right)$.

Proof. By (9) it is obvious that $\omega_{A}\left(\phi_{1}\right) \omega_{A}^{*}\left(\phi_{1}\right)=\Omega\left(\phi_{1} \phi_{1}^{*}\right)$ and $\omega_{\Lambda}\left(\phi_{1}\right) \omega_{A}^{*}\left(\phi_{2}\right)$ 
$=\Omega\left(\phi_{1} \phi_{2}^{*}\right)$. Therefore, since we can assume $\phi=\alpha \phi_{1}, \psi=\beta_{1} \phi_{1}+\beta_{2} \phi_{2}$, the first assertion follows by linearity.

Similarly, $\omega_{A}^{*}\left(\phi_{1}\right) \omega_{A}\left(\phi_{1}\right)=I-\Omega\left(\phi_{1} \phi_{1}^{*}\right), \omega_{A}^{*}\left(\phi_{1}\right) \omega_{A}\left(\phi_{2}\right)=-\Omega\left(\phi_{2} \phi_{1}^{*}\right)$, and the second assertion also follows by linearity.

Define $[A, B]_{+}=A B+B A$. Then:

Theorem 6. $\left[\omega_{A}(\phi), \omega_{A}^{*}(\psi)\right]_{+}=(\phi, \psi) I,\left[\omega_{A}(\phi), \omega_{A}(\psi)\right]_{+}=\left[\omega_{A}^{*}(\phi), \omega_{A}^{*}(\psi)\right]_{+}$ $=0$.

Proof. Use Lemma 1 on the first expression and (9) on the others.

THEOREM 7. If $\left\{\phi_{i}\right\}$ is an orthonormal basis of $\Re$, then the set $\left\{\omega_{A}\left(\phi_{i}\right)\right\}$ is irreducible on $\mathfrak{A}$.

Proof. Let $E$ be a projection such that $E \omega_{A}\left(\phi_{i}\right)=\omega_{A}\left(\phi_{i}\right) E$ for all $i$. Then we must prove $E=0$ or $I[8$, p. 33].

$E \omega_{A}\left(\phi_{i}\right) \omega_{A}^{*}\left(\phi_{i}\right)=\left(\omega_{A}\left(\phi_{i}\right) \omega_{A}^{*}\left(\phi_{i}\right) E\right.$ and $E \omega_{A}^{*}\left(\phi_{i}\right) \omega_{A}\left(\phi_{i}\right)=\omega_{A}^{*}\left(\phi_{i}\right) \omega_{A}\left(\phi_{i}\right) E$. But, by Lemma $1, \omega_{A}\left(\phi_{i}\right) \omega_{A}^{*}\left(\phi_{i}\right)=\Omega\left(\phi_{i} \phi_{i}^{*}\right)$ the projection of $\mathfrak{A}$ on the subspace spanned by basis elements of the form $\left(\phi_{1}^{n_{1}} \phi_{2}^{n_{1}} \cdots\right)_{A}$ with $n_{i}=1$; and $\omega_{A}^{*}\left(\phi_{i}\right) \omega_{A}\left(\phi_{i}\right)=I-\Omega\left(\phi_{i} \phi_{i}^{*}\right)$ the projection of $\mathfrak{A}$ on the subspace spanned by such basis elements with $n_{i}=0$. The set of all these projections generates the maximal abelian algebra of all normal operators for which every $\left(\phi_{1}^{n_{1}} \phi_{2}^{n_{2}} \cdots\right)_{A}$ is an eigenvector. But, by (9), the only such subspace invariant under all $\omega_{A}\left(\phi_{i}\right), \omega_{A}^{*}\left(\phi_{j}\right)$ is either 0 or $\mathfrak{A}$.

4. To treat the symmetric case, we restrict all our operators to the subspace $\mathfrak{S}=\left(\sum_{n=0}^{\infty} \oplus P_{s_{n}}\right) \mathfrak{F}$ and define $\omega_{G}$ and $\omega_{G}{ }^{*}$ by picking $G$ to be that particular function $S$ which assigns to every non-negative integer $n$ the operator $n^{1 / 2} P_{s_{n}}$ in $G_{n}$. Thus $\omega_{s}(\phi)=\left(\sum_{n=0}^{\infty} \oplus n^{1 / 2} P_{s_{n}}\right)(\phi \otimes)$ and $\omega_{s}^{*}(\phi)=\left((\phi \otimes)^{*}\left(\sum_{n=0}^{\infty}\right.\right.$ $\left.\left.\oplus n^{1 / 2} P_{s_{n}}\right)\right)^{\sim}$ considered as linear transformations of $\mathcal{S}$. $\mathcal{S}$ reduces $\Omega(A)$ so the discussions in $\S \S 1$ and 2 apply unchanged.

For any orthonormal basis $\left\{\phi_{i}\right\}$ of $\Re$, we define an orthonormal basis $\left\{\left(\phi_{1}^{n_{n}} \phi_{2}^{n_{2}} \cdots\right)_{s}\right\}$ of $\mathfrak{S}$, where $n=0,1,2, \cdots$ and $\sum_{i} n_{i}<\infty$, by

$$
\begin{aligned}
& \left(\phi_{1}^{n_{1} \phi_{2} n_{2}} \cdots\right)_{s} \\
& \quad=\mathrm{i}\left(\frac{\left(\sum_{i} n_{i}\right) !}{\prod_{i}\left(n_{i} !\right)}\right)^{1 / 2} P_{s \Sigma n i} \phi_{1} \otimes \cdots \otimes \phi_{1} \otimes \phi_{2} \otimes \cdots \otimes \phi_{2} \otimes \phi_{3} \otimes \cdots
\end{aligned}
$$

(Each $\phi_{i}$ appears $n_{i}$ times in the tensor product.)

For any subset $\mathfrak{N}$ of $\mathfrak{R}$, let $[\mathfrak{R}$ ] be the closed linear manifold which it generates; and for any closed linear manifold $\mathfrak{M}$ of $\Re$, let $P_{\mathfrak{M}}$ be the projection on that manifold. Then

LEMMA 2. $\mathfrak{D}_{\omega_{\theta}(\phi)}=\mathfrak{D}_{\omega_{\theta}^{*}(\phi)}=\mathfrak{D}_{(\Omega(P[\phi]))^{1 / 2}}$. If $f=\sum \alpha_{n_{1} n_{2}} \ldots\left(\phi_{1}^{n_{1}} \phi_{2}^{n_{2}} \cdots\right)$. $\in \mathfrak{D}_{\omega_{0}\left(\phi_{i}\right)}$, then $\omega_{s}\left(\phi_{i}\right) f=\sum \alpha_{n_{1} n_{2}} \ldots\left(n_{i}+1\right)^{1 / 2}\left(\phi_{1}^{n_{1}} \phi_{2}^{n_{2}} \cdots \phi_{i}^{n_{i}+1}\right)$, and $\omega_{s}^{*}\left(\phi_{i}\right) f$ $=\sum \alpha_{n_{1} n_{2}} \cdots n_{i}^{1 / 2}\left(\phi_{1}^{n_{1}} \phi_{2}^{n_{n}} \cdots \phi_{i}^{n_{i}-1} \cdots\right)_{*}$. 
Proof. By a straightforward manipulation of the definitions it can be seen that $\omega_{s}\left(\phi_{i}\right)\left(\phi_{1}^{n_{1}} \phi_{2}^{n_{2}} \cdots\right)_{s}=\left(n_{i}+1\right)^{1 / 2}\left(\phi_{1}^{n_{1}} \phi_{2}^{n_{2}} \cdots \phi_{i}^{n_{i}+1} \cdots\right)_{s}$ and $\omega_{s}^{*}\left(\phi_{i}\right)\left(\phi_{1}^{n_{1}} \phi_{2}^{n_{1}} \cdots\right)_{s}=n_{i}^{1 / 2}\left(\phi_{1}^{n_{1}} \phi_{2}^{n_{n}} \cdots \phi_{i}^{n_{i}-1} \cdots\right)_{s}$ (making the convention $\left(\phi_{1}^{n_{1}} \phi_{2}^{n_{2}} \cdots\right)_{s}=0$ if $\left.n_{i}<0\right)$. Therefore, since $\omega_{s}\left(\phi_{i}\right)$ and $\omega_{s}^{*}\left(\phi_{i}\right)$ are closed, $f \in \mathfrak{D}_{\omega_{\varepsilon}\left(\phi_{i}\right)}$ if and only if $\sum\left(n_{i}+1\right)\left|\alpha_{n_{1} n_{2}} \cdots\right|^{2}<\infty$, in which case $\omega_{s}\left(\phi_{i}\right) f$ $=\sum \alpha_{n_{1} n_{2}} \ldots\left(n_{i}+1\right)^{1 / 2}\left(\phi_{1}^{n_{1}} \phi_{2}^{n_{2}} \cdots \phi_{i}^{n_{i}+1} \cdots\right)_{s}$; and $f \in \mathcal{D}_{\omega_{s}^{*}\left(\phi_{1}\right)}$ if and only if $\sum n_{i}\left|\alpha_{n_{1} n_{2}} \ldots\right|^{2}<\infty$, in which case $\omega_{8}^{*}\left(\phi_{i}\right) f=\sum \alpha_{n_{1} n_{2}} \ldots n_{i}{ }^{1 / 2}\left(\phi_{1}^{n_{1}} \phi_{2}^{n_{2}} \ldots\right.$ $\left.\phi_{i}^{n_{i}-1} \ldots\right)_{s}$.

Since $\sum^{s}\left(n_{1}+1\right)\left|\alpha_{n_{1} n_{2}} \ldots\right|^{2}=\|f\|^{2}+\sum n_{1}\left|\alpha_{n_{1} n_{2}} \ldots\right|^{2}$, it is easily seen (let $\phi_{1}$ $=(1 /\|\phi\|) \phi)$ that if $\phi$ is any element of $\Re$ then $\left\|\omega_{s}(\phi) f\right\|^{2}=\left\|\omega_{s}^{*}(\phi) f\right\|^{2}$ $+\|\phi\|^{2}\|f\|^{2}$, so $\mathfrak{D}_{\omega_{\varepsilon}(\phi)}=\mathfrak{D}_{\omega_{s}^{*}(\phi)}$. Further, since $\left(\Omega\left(P_{\left[\phi_{1}\right]}\right)\right)^{1 / 2}\left(\phi_{1}^{n_{1}} \phi_{2}^{n_{1}} \cdots\right)_{s}$ $=n_{1}^{1 / 2}\left(\phi_{1}^{n_{1}} \phi_{2}^{n_{n}} \cdots\right)_{s}=\omega_{s}\left(\phi_{1}\right)\left(\phi_{1}^{n_{1}-1} \phi_{2}^{m_{n}} \cdots\right)_{s}$, the only remaining assertion is obvious.

Let $V_{\phi_{1}}$ be the bounded linear extension of the transformation $V_{\phi_{1}}\left(\phi_{1}^{n_{1}} \phi_{2}^{n_{2}} \cdots\right)_{s}=\left(\phi_{1}^{n_{1}+1} \phi_{2}^{n_{2}} \cdots\right)_{s}$ of $\mathfrak{S}$. Then $V_{\phi_{1}}^{*}$ is the bounded linear extension of the transformation $V_{\phi_{1}}^{*}\left(\phi_{1}^{n_{1}} \phi_{2}^{n_{2}} \cdots\right)_{s}=\left(\phi_{1}^{n_{1}-1} \phi_{2}^{n_{1}} \cdots\right)_{s} . V_{\phi_{1}}$ is an isometry of the range of $\left(\Omega\left(P_{\left[\phi_{1}\right]}\right)\right)^{1 / 2}$. The polar decomposition [8, p. 53] $\omega_{s}\left(\phi_{1}\right)=V_{\phi_{1}}\left(\Omega\left(P_{\left[\phi_{1}\right]}\right)+I\right)^{1 / 2}, \quad \omega_{s}^{*}\left(\phi_{1}\right)=V_{\phi_{1}}^{*}\left(\Omega\left(P_{\left[\phi_{1}\right]}\right)\right)^{1 / 2}$ of $\omega_{s}\left(\phi_{1}\right)$ and $\omega_{s}^{*}\left(\phi_{1}\right)$, and therefore of $\omega_{s}(\phi), \omega_{s}^{*}(\phi)$ for arbitrary $\phi$, follows immediately from the preceding lemma.

For this symmetric case we can supplement Theorem 5 by the statement: $\left[\Omega(A), \omega_{s}(\phi)\right]$ is closed if and only if $A=0$ and $\phi=0 ;\left[\Omega(A), \omega_{s}^{*}(\psi)\right]$ if and only if $A=0$ and $\psi=0$.

Proof. Assume that $\left[\Omega(A), \omega_{s}(\phi)\right]$ is closed, i.e., that $\mathfrak{D}_{\left[\Omega(A), \omega_{s}(\phi)\right]}$ $=\mathfrak{D}_{\omega_{s}(A \phi)}$. Then $\phi$ must be an eigenvector of $A$ since otherwise, by Lemma 2, $\mathfrak{D}_{\omega_{\alpha}(A \phi)}$ is not contained in $\mathfrak{D}_{\omega_{\mathcal{A}}(\phi)}$. If $A \phi=\lambda \phi$ with $\lambda \phi \neq 0$, then for $g=\sum_{n=1}^{\infty}$ $\oplus\left(1 / n^{3 / 2}\|\phi\|^{n}\right) \phi \otimes \cdots \otimes \phi\left(n \phi^{\prime} \mathrm{s}\right)$ we have $\|g\|^{2}=\sum_{n=1}^{\infty}\left(1 / n^{3}\right)<\infty,\left\|\omega_{s}(A \phi) g\right\|^{2}$ $=\|\lambda \phi\|^{2} \sum_{n=1}^{\infty}(n+1) / n^{3}<\infty,\|\Omega(A) g\|^{2}=|\lambda|^{2} \sum_{n=1}^{\infty} 1 / n=\infty$. So $g \in \mathcal{D}_{\omega_{\mathrm{s}}(A \phi)}$ but $g \notin \mathfrak{D}_{\Omega(A)} \supseteq \mathfrak{D}_{\left[\Omega(A), \omega_{s}(\phi)\right]}$. This contradiction implies $A \phi=0$. But then $\mathfrak{S}=\mathfrak{D}_{\omega_{\mathcal{g}}(A \phi)}=\mathfrak{D}_{\left[\Omega(A), \omega_{\varepsilon}(\phi)\right]}=\mathfrak{D}_{\mathbb{Q}(A)}$, so $A=0$ by the proof of Theorem 2(e). Also $\mathfrak{D}_{\omega_{s}(\phi)}=\mathfrak{S}$, so $\phi=0$.

The second assertion is proved similarly, and both converses are obvious.

Lemma 3. $\omega_{s}(\psi) \omega_{s}^{*}(\phi)$ and $\omega_{s}^{*}(\psi) \omega_{s}(\phi)$ are densely defined linear operators with closures $\left(\omega_{s}(\psi) \omega_{s}^{*}(\phi)\right)^{\sim}=\Omega\left(\psi \phi^{*}\right),\left(\omega_{s}^{*}(\psi) \omega_{s}(\phi)\right)^{\sim}=(\phi, \psi) I+\Omega\left(\phi \psi^{*}\right)$, to which the first is equal if and only if $\psi \neq 0$ or $\phi=0$, the second if and only if $\phi=\lambda \psi$.

Proof. $\omega_{s}(\psi) \omega_{s}^{*}(\phi)$ and $\omega_{s}^{*}(\psi) \omega_{s}(\phi)$ are obviously densely defined linear operators. Since the lemma is trivial when $\phi=0$, we may assume that $\left\{\phi_{i}\right\}$ is an orthonormal basis of $\Re$ such that $\phi=\phi_{1}, \psi=\alpha_{1} \phi_{1}+\alpha_{2} \phi_{2}$. Then $\omega_{s}(\psi) \omega_{s}^{*}(\phi)\left(\phi_{1}^{n_{1}} \phi_{2}^{n_{n}} \cdots\right)_{s}=n_{1}^{1 / 2} \omega_{s}(\psi)\left(\phi_{1}^{n_{1}-1} \phi_{2}^{n_{2}} \cdots\right)_{s}=\alpha_{1} n_{1}\left(\phi_{1}^{n_{1}} \phi_{2}^{n_{2}} \cdots\right)_{s}$ $+\alpha_{2}\left(n_{1}\left(n_{2}+1\right)\right)^{1 / 2}\left(\phi_{1}^{n_{1}-1} \phi_{2}^{n_{n}+1} \ldots\right)_{s}=\Omega\left(\psi \phi^{*}\right)\left(\phi_{1}^{n_{1}} \phi_{2}^{n_{2}} \ldots\right)_{s}$ so $\omega_{s}(\psi) \omega_{s}^{*}(\phi)$ and $\Omega\left(\psi \phi^{*}\right)$ agree on an orthonormal basis of each $\mathfrak{S}^{(n)}=\left(O \otimes \cdots \oplus O \oplus I^{(n)}\right.$ 
$\oplus O \oplus \ldots) \subseteq$. Since they are both bounded when restricted to $\mathfrak{S}^{(n)}$, they must agree all over $\mathfrak{S}^{(n)}$. Now let $f=\sum_{n=0}^{\infty} f_{n} \in \mathfrak{D}_{\omega_{0}(\psi) \omega_{s}^{*}(\phi)}, f_{n} \in \mathbb{S}^{(n)}$. Then $\omega_{s}^{*}(\phi) f=\sum_{n=0}^{\infty} \omega_{s}^{*}(\phi) f_{n}$ and $\omega_{s}(\psi) \omega_{s}^{*}(\phi) f=\sum_{n=0}^{\infty} \omega_{s}(\psi) \omega_{s}^{*}(\phi) f_{n}=\sum_{n=0}^{\infty} \Omega\left(\psi \phi^{*}\right) f_{n}$ $=\Omega\left(\psi \phi^{*}\right) f$, so $f \in \mathcal{D}_{\Omega\left(\psi \phi^{*}\right)}$ and $\omega_{s}(\psi) \omega_{s}^{*}(\phi) f=\Omega\left(\psi \phi^{*}\right) f, \omega_{s}(\psi) \omega_{s}^{*}(\phi) \subseteq \Omega\left(\psi \phi^{*}\right)$.

Since $\omega_{s}(\psi) \omega_{\varepsilon}^{*}(\phi)$ has the closed linear extension $\Omega\left(\psi \phi^{*}\right)$, it has a closure $\left(\omega_{s}(\psi) \omega_{s}^{*}(\phi)\right)^{2} \subseteq \Omega\left(\psi \phi^{*}\right)$. If $f \in \mathfrak{D}_{\Omega\left(\psi \phi^{*}\right)}, f=\lim _{m \rightarrow \infty} \sum_{n=0}^{m} f_{n}, f_{n} \in \mathfrak{S}^{(n)}$, then $\sum_{n=0}^{m} f_{n} \in \mathfrak{D}_{\left(\omega_{s}(\psi) \omega_{s}^{*}(\phi)\right)} \sim$ and $\lim _{m \rightarrow \infty}\left(\omega_{s}(\psi) \omega_{s}^{*}(\phi)\right)^{\sim} \sum_{n=0}^{m} f_{n}=\Omega\left(\psi \phi^{*}\right) f$, so $\in \mathfrak{D}_{\left(\omega_{s}(\psi) \omega_{*}^{*}(\phi)\right)} \sim$ and $\left(\omega_{s}(\psi) \omega_{s}^{*}(\phi)\right)^{\sim} f=\Omega\left(\psi \phi^{*}\right) f .\left(\omega_{s}(\psi) \omega_{s}^{*}(\phi)\right)^{\sim}=\Omega\left(\psi \phi^{*}\right)$.

If $\psi=0$, then $\mathfrak{D}_{\omega_{s}(\psi) \omega_{*}^{*}(\phi)}=\mathfrak{D}_{\omega_{s}^{*}(\phi)} \neq S=\mathfrak{D}_{\Omega(0)}=\mathfrak{D}_{\Omega\left(\psi \phi^{*}\right)}$, so $\omega_{s}(\psi) \omega_{s}^{*}(\phi)$ is not closed. Conversely, if $\psi \neq 0$, then $\omega_{s}(\psi) \omega_{s}^{*}(\phi)=\|\psi\| V_{\psi /\|\psi\|}\left(\Omega\left(P_{[\psi]}\right)\right.$ $+I)^{1 / 2} \omega_{s}{ }^{*}(\phi)$ is the product of an isometry, of a transformation with a bounded inverse, and of a closed transformation in that order. Therefore, as can be seen from the definition of a closed operator, it is closed.

The discussion of $\omega_{s}{ }^{*}(\psi) \omega_{s}(\phi)$ is similar. As before, assume $\left\{\phi_{i}\right\}$ an orthonormal basis of $\Re, \phi=\phi_{1}$, and $\psi=\alpha_{1} \phi_{1}+\alpha_{2} \phi_{2}$. Then $\omega_{s}^{*}(\psi) \omega_{s}(\phi)\left(\phi_{1}^{n_{1}} \phi_{2}^{n_{1}} \cdots\right)_{s}$ $=\omega_{s}^{*}(\psi)\left(n_{1}+1\right)^{1 / 2}\left(\phi_{1}^{n_{1}+1} \phi_{2}^{n_{2}} \ldots\right)_{s}=\alpha_{1}^{*}(n+1)\left(\phi_{1}^{n_{1}} \phi_{2}^{n_{2}} \ldots\right)_{s}+\alpha_{2}^{*}\left(\left(n_{1}+1\right) n_{2}\right)^{1 / 2}$ $\cdot\left(\phi_{1}^{n_{1}+1} \phi_{2}^{n_{1}-1} \cdots\right)_{s}=\alpha_{1}^{*} I\left(\phi_{1}^{n_{1}} \phi_{2}^{n_{n}} \cdots\right)_{s}+\left(\alpha_{1}^{*} \Omega\left(\phi_{1} \phi_{1}^{*}\right)+\alpha_{2}^{*} \Omega\left(\phi_{1} \phi_{2}^{*}\right)\right)\left(\phi_{1}^{n_{1}} \phi_{2}^{n_{2}} \cdots\right)_{s}$ $=\left(\left(\psi, \phi_{1}\right)^{*} I+\Omega\left(\phi_{1} \alpha_{1}^{*} \phi_{1}^{*}+\phi_{1} \alpha_{2}^{*} \phi_{2}^{*}\right)\right)\left(\phi_{1}^{n_{1}} \phi_{2}^{n_{2}} \cdots\right)_{s}=\left((\phi, \psi) I+\Omega\left(\phi \psi^{*}\right)\right)$ $\cdot\left(\phi_{1}^{n_{1}} \phi_{2}^{n_{2}} \ldots\right)_{s}$, so $\omega_{s}^{*}(\psi) \omega_{s}(\phi)$ and $(\phi, \psi) I+\Omega\left(\phi \psi^{*}\right)$ agree on an orthonormal basis. Therefore, as in the previous case, $\omega_{s}^{*}(\psi) \omega_{s}(\phi) \subseteq(\phi, \psi) I+\Omega\left(\phi \psi^{*}\right)$ and $\left(\omega_{s}^{*}(\psi)\left(\omega_{s}(\phi)\right)^{\sim}=(\phi, \psi) I+\Omega\left(\phi \psi^{*}\right)\right.$.

Now assume $\phi=\lambda \psi$. If $\phi=0$ then $\omega_{s}^{*}(\psi) \omega_{s}(\phi)$ is closed, so assume $\|\phi\|=1$. Then $\omega_{s}^{*}(\psi) \omega_{s}(\phi)=\lambda^{*-1} \omega_{s}^{*}(\phi) \omega_{s}(\phi)=\lambda^{*-1} V_{\phi}^{*}\left(\Omega\left(P_{[\phi]}\right)\right)^{1 / 2} V_{\phi}\left(\Omega\left(P_{[\phi]}\right)+I\right)^{1 / 2}$ $=\lambda^{*-1}\left(\Omega\left(P_{[\phi]}\right)+I\right)^{1 / 2} V_{\phi}^{*} V_{\phi}\left(\Omega\left(P_{[\phi]}\right)+I\right)^{1 / 2}=\lambda^{*-1}\left(\Omega\left(P_{[\phi]}\right)+I\right)$, which is closed.

Conversely assume $\phi \notin[\psi]$. Then $\phi \neq 0$ and, since $\psi=0$ implies $\mathfrak{D}_{\omega_{\theta}^{*}(\psi) \omega_{s}(\phi)}$ $=\mathfrak{D}_{\omega_{s}(\phi)} \neq \mathfrak{S}=\mathfrak{D}_{0}=\mathfrak{D}_{(\phi, \psi) I+\Omega\left(\phi \psi^{*}\right)}=\mathfrak{D}_{\left(\omega_{\varepsilon}^{*}(\psi) \omega_{s}(\phi)\right)} \sim$, i.e., implies $\omega_{s}^{*}(\psi) \omega_{\varepsilon}(\phi)$ not closed, we may also assume $\psi \neq 0$. Now we need only construct a $g$ in $\mathfrak{D}_{\left(\omega_{\theta}^{*}(\psi) \omega_{\theta}(\phi)\right.} \sim$ such that $g \in D_{\omega_{0}^{*}(\psi) \omega_{\theta}(\phi)}$. Let $\phi^{\prime}=\phi-\left((\phi, \psi) /\|\psi\|^{2}\right) \psi$ and $\phi_{0}$ $=\left(1 /\left\|\phi^{\prime}\right\|\right) \phi^{\prime}$. Then $\left(\phi \psi^{*}\right) \phi_{0}=0$ and, if we let $g=0 \oplus \sum_{n=1}^{\infty} \oplus(1 / n) \phi_{0} \otimes \ldots$ $\otimes \phi_{0}$, then $\Omega\left(\phi \psi^{*}\right) g=0, g \in \mathfrak{D}_{\Omega\left(\phi \psi^{*}\right)}=\mathfrak{D}_{(\phi, \psi) I+\Omega\left(\phi \psi^{*}\right)}=\mathfrak{D}_{\left(\omega_{\theta^{*}}(\psi) \omega_{8}(\phi)\right)} \sim$. But $\phi=\beta_{0} \phi_{0}$ $+\beta_{1} \psi$, with $\beta_{0} \neq 0$, so $\omega_{s}(\phi) g=0 \oplus \sum_{n=1}^{\infty} \oplus\left(1 / n(n+1)^{1 / 2}\right)\left\{\phi \otimes \phi_{0} \otimes \cdots \otimes \phi_{0}\right.$ $\left.+\phi_{0} \otimes \phi \otimes \cdots \otimes \phi_{0}+\cdots\right\}$ ( $\phi_{0}$ appears $n$ times in each tensor product) $=0 \oplus \sum_{n=1}^{\infty} \oplus\left[\left(1 / n(n+1)^{1 / 2}\right)\left\{\beta_{0}(n+1) \phi_{0} \otimes \cdots \otimes \phi_{0}\right\}+\psi_{n+1}\right]$, where the tensor product $\phi_{0} \otimes \cdots \otimes \phi_{0}$ of $\phi_{0}$ with itself $n+1$ times is orthogonal to $\psi_{n+1}$ in $\Re^{(n+1)}$. Therefore $\left\|\omega_{s}(\phi) g\right\|^{2}=\left|\beta_{0}\right|^{2} \sum_{n=1}^{\infty}\left\{\left((n+1) / n^{2}\right)+\left\|\psi_{n+1}\right\|^{2}\right\}$ $\geqq\left|\beta_{0}\right|^{2} \sum_{n=1}^{\infty}(1 / n)=\infty, g \in \mathfrak{D}_{\omega_{s}(\phi)} \supseteq \mathfrak{D}_{\omega_{\varepsilon}}^{*}(\psi) \omega_{s}(\phi)$.

THEOREM 8. The brackets $\left[\omega_{s}(\phi), \omega_{s}(\psi)\right],\left[\omega_{s}^{*}(\phi), \omega_{s}^{*}(\psi)\right],\left[\omega_{s}^{*}(\phi), \omega_{s}(\psi)\right]$ are densely defined, linear transformations with the closures $\left(\left[\omega_{s}(\phi), \omega_{s}(\psi)\right]\right)$ $=0,\left(\left[\omega_{s}^{*}(\phi), \omega_{s}^{*}(\psi)\right]\right)^{\sim}=0,\left(\left[\omega_{s}^{*}(\phi), \omega_{s}(\psi)\right]\right)^{\mathcal{\nu}}=(\psi, \phi) I$ to which they are equal if and only if $\phi=\psi=0$.

(Using Lemma 3 on the third expression, the proof is an easy repetition of methods already used several times.) 
THEOREM 9. If $\left\{\phi_{i}\right\}$ is an orthonormal basis of $\Re$, then the set $\left\{\omega_{s}\left(\phi_{i}\right)\right\}$ is irreducible on 5 .

Proof. Assume $E$ to be a projection such that $E \omega_{s}\left(\phi_{i}\right) \subseteq \omega_{s}\left(\phi_{i}\right) E$ for all $i[8$, pp. 32-33]. Then $E \omega_{s}^{*}\left(\phi_{i}\right) \subseteq \omega_{s}^{*}\left(\phi_{i}\right) E$ also, and $E \omega_{s}\left(\phi_{i}\right) \omega_{s}^{*}\left(\phi_{i}\right) \subseteq \omega_{s}\left(\phi_{i}\right) \omega_{s}^{*}\left(\phi_{i}\right) E$. But $\omega_{s}\left(\phi_{i}\right) \omega_{s}^{*}\left(\phi_{i}\right)=\Omega\left(\phi_{i} \phi_{i}^{*}\right)=\Omega\left(P_{\left[\phi_{1}\right.}^{i}\right)$ by Lemma 3 , and projections arising from the spectral decompositions of the $\Omega P\left({ }_{\left[\phi_{i}\right]}\right)$ generate the maximal abelian algebra of all normal operators for which every $\left(\phi_{1}^{n_{1}} \phi_{2}^{n_{n}} \cdots\right)_{s}$ is an eigenvector. Therefore $E S$ is spanned by some subset of these eigenvectors. But, by Lemma 2 , the only such subspace invariant under all $\omega_{s}\left(\phi_{i}\right), \omega_{s}^{*}(\phi$,$) is either$ 0 or $\subseteq$ itself.

DEFINITION 6.

$$
p(\phi)=i\left(\left[\omega_{s}(\phi)-\omega_{s}^{*}(\phi)\right]\right) \sim / 2^{1 / 2}, \quad q(\phi)=\left(\left[\omega_{s}(\phi)+\omega_{s}^{*}(\phi)\right]\right) \sim / 2^{1 / 2} .
$$

This definition implies that the operators have closures, a fact contained in the following theorem:

\section{THEOREM 10. $p(\phi)$ and $q(\phi)$ are self-adjoint.}

Proof. Since $q(\phi)=p(-i \phi)$, we need only consider $p(\phi)$. Further, since $p(\phi)=\|\phi\| p\left(\phi_{1}\right)$, where $\left\|\phi_{1}\right\|=1$, we can assume that $\phi=\phi_{1}$ an element of the orthonormal basis $\left\{\phi_{i}\right\}$ of $\Re$. $\mathfrak{D}_{\omega_{e}\left(\phi_{1}\right)}=\mathfrak{D}_{\omega_{e}^{*}\left(\phi_{1}\right)}=\mathfrak{D}_{i\left[\omega_{e}\left(\phi_{1}\right)-\omega_{\dot{\theta}}^{*}\left(\phi_{1}\right)\right]}$ is dense in S, so $\left(i\left[\omega_{s}\left(\phi_{1}\right)-\omega_{s}^{*}\left(\phi_{1}\right)\right]\right)^{*}$ exists and $\left(i\left[\omega_{s}\left(\phi_{1}\right)-\omega_{s}^{*}\left(\phi_{1}\right)\right]\right)^{*}=-i\left[\omega_{s}\left(\phi_{1}\right)\right.$ $\left.-\omega_{s}^{*}\left(\phi_{1}\right)\right]^{*} \supseteq-i\left[\omega_{s}^{*}\left(\phi_{1}\right)-\omega_{s}\left(\phi_{1}\right)\right]=-i\left[\omega_{s}^{*}\left(\phi_{1}\right)-\omega_{s}\left(\phi_{1}\right)\right]=i\left[\omega_{s}\left(\phi_{1}\right)-\omega_{s}^{*}\left(\phi_{1}\right)\right]$, i.e. $i\left[\omega_{s}\left(\phi_{1}\right)-\omega_{s}^{*}\left(\phi_{1}\right)\right]$ is symmetric $[8$, p. 34$]$, and hence has a closure; so $p(\phi)$ and $q(\phi)$ exist.

$i\left(\left[\omega_{s}\left(\phi_{1}\right)-\omega_{s}^{*}\left(\phi_{1}\right)\right]\right)^{\sim}$ is self-adjoint if and only if its deficiency indices are $(0,0)[8$, p. 38]. Since it is real $[8$, p. 40] on the orthonormal basis $\left\{i^{n_{1}}\left(\phi_{1}^{n_{1}} \phi_{2}^{n_{2}} \cdots\right)_{s}\right\}$, its deficiency indices have the form $(m, m)$, and we need only prove that the single deficiency space $\mathfrak{D}^{-}$is zero-dimensional, where $\mathfrak{D}^{-}$is the set of all $g$ in $\subseteq$ which are orthogonal to $\left(i\left[\omega_{s}\left(\phi_{1}\right)-\omega_{s}^{*}\left(\phi_{1}\right)\right]\right.$ $-i I) \mathfrak{D}_{i\left[\omega_{s}\left(\phi_{1}\right)-\omega_{s}^{*}\left(\phi_{1}\right)\right]} . \quad$ But if $\sum g_{m_{1} m_{2}} \ldots\left(\phi_{1}^{m_{1}} \phi_{2}^{m_{2}} \cdots\right)_{s}=g \in \mathfrak{D}^{-}$, then 0 $=\left(g, \quad\left(i\left[\omega_{s}\left(\phi_{1}\right)-\omega_{s}^{*}\left(\phi_{1}\right)\right]-i I\right)\left(\phi_{1}^{n_{1} \phi_{2}^{n_{2}} \cdots}\right)_{s}\right)=\left(g, \quad i\left(n_{1}+1\right)^{1 / 2}\left(\phi_{1}^{n_{1}+1} \phi_{2}^{n_{2}} \cdots\right)_{s}\right.$ $\left.-i n_{1}^{1 / 2}\left(\phi_{1}^{n_{1}-1} \phi_{2}^{n_{1}} \ldots\right)_{s}-i\left(\phi_{1}^{n_{1}} \phi_{2}^{n_{2}} \cdots\right)_{s}\right)=-i g_{n_{1}+1, n_{2}}, \ldots\left(n_{1}+1\right)^{1 / 2}+i g_{n_{1}-1, n_{2}}, \ldots$ $n_{1}^{1 / 2}+i g_{n_{1}, n_{2}}, \ldots$ (making the convention $g_{-1, n_{2}, n_{3}}, \ldots=0$ ). Therefore we have the recurrence formula $\left(n_{1}+1\right)^{1 / 2} g_{n_{1}+1, n_{2}}, \ldots=g_{n_{1}, n_{2}}, \ldots+n_{1}^{1 / 2} g_{n_{1}-1, n_{2}}, \ldots$ In particular, $g_{0, n_{2}, \ldots}=g_{1, n_{2}, \ldots .}$

If for some $n_{2}, n_{3}, \cdots$ we have $g_{0, n_{2}}, \ldots \neq 0$, let $\tilde{g}=\left(1 / g_{0, n_{2}}, \ldots\right) g$. Then $\tilde{g}_{0, n_{2}}, \ldots=\tilde{g}_{1, n_{2}}, \ldots=1$. But, from the recurrence formula, $\tilde{g}_{n_{1}-1, n_{2}}, \ldots \geqq 1$, $\tilde{g}_{n_{1}, n_{2}}, \ldots \geqq 1 \quad$ implies $\quad \tilde{g}_{n_{1}+1, n_{2}}, \ldots=\left(\tilde{g}_{n_{1}, n_{2}}, \ldots+n_{1}^{1 / 2} \tilde{g}_{n_{1}-1, n_{2}}, \ldots\right) /\left(n_{1}+1\right)^{1 / 2} \geqq(1$ $\left.+n_{1}^{1 / 2}\right) /\left(n_{1}+1\right)^{1 / 2} \geqq 1$. Hence, $\tilde{g}_{k, n_{2}}, \ldots \geqq 1$ for all $k=0,1,2, \cdots$, contradict-

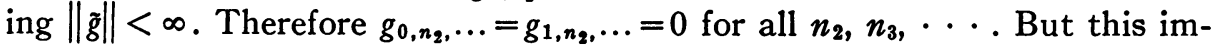
plies, by another easy induction using the recurrence formula, that $g_{n_{1}, n_{2}}, \ldots$ $=0$ for all $n_{1}, n_{2}, \cdots$, and so $g=0, \mathfrak{D}^{-}=0$. 
THEOREM 11. The brackets $[q(\phi), q(\psi)],[q(\phi), p(\psi)]$, and $[p(\phi), p(\psi)]$ are densely defined, linear operators with the closures $([q(\phi), q(\psi)])^{\sim}=((\psi, \phi)$ $-(\phi, \psi)) I / 2,([q(\phi), p(\psi)])^{\sim}=i((\psi, \phi)-(\phi, \psi)) I / 2,([p(\phi), p(\psi)])^{\sim}=((\psi, \phi)$ $-(\phi, \psi)) I / 2$ to which they are equal if and only if $\phi=\psi=0$.

Proof. Since $p(\phi)=q(i \phi)$, we need only consider the bracket $[q(\phi), q(\psi)]$.

Let $g \in \mathfrak{D}_{[q(\phi), q(\psi)]}$. Then $\left(\left(\phi_{1}^{n_{1}} \phi_{2}^{n_{2}} \cdots\right)_{s}, \quad[q(\phi), \quad q(\psi)] g\right)=\left(\left[\left(\omega_{s}(\psi)\right.\right.\right.$ $\left.\left.\left.+\omega_{s}^{*}(\psi)\right) / 2^{1 / 2}, \quad\left(\omega_{s}(\phi)+\omega_{s}^{*}(\phi)\right) / 2^{1 / 2}\right]\left(\phi_{1}^{n_{1}} \phi_{2}^{n_{1}} \ldots\right)_{s}, \quad g\right)=((1 / 2)((\phi, \quad \psi)-(\psi$, $\left.\phi))\left(\phi_{1}^{n_{1}} \phi_{2}^{n_{3}} \cdots\right)_{8}, \quad g\right)=\left(\left(\phi_{1}^{n_{1}} \phi_{2}^{n_{2}} \cdots\right)_{8}, \quad(1 / 2)((\psi, \quad \phi)-(\phi, \psi)) g\right)$, so

$$
[q(\phi), q(\psi)] \subseteq((\psi, \phi)-(\phi, \psi)) I / 2 .
$$

Lemma 4. If $A$ is a self-adjoint operator on $\Re$ and $\phi \in \mathfrak{D}_{A}$, then $i([\Omega(A)$, $p(\phi)])^{\sim}=p(i A \phi)$ and $i([\Omega(A), q(\phi)])^{\sim}=q(i A \phi)$.

Proof. $i([\Omega(A), q(\phi)])^{\sim} \supseteq i\left[\Omega(A),\left(\omega_{s}(\phi)+\omega_{s}^{*}(\phi)\right) / 2^{1 / 2}\right] \supseteq q_{0}(i A \phi)$ by Theorem 5, where $q_{0}(i A \phi)$ is $q(i A \phi)$ restricted to the domain of all $\sum_{n=0}^{\infty} \oplus f_{n}$ in $\subseteq$ such that $\mathrm{i} f_{n} \in \mathfrak{D}_{\Omega(A)}$ for $n=0,1,2, \cdots$ and only a finite number of the $f_{n}$ are nonzero. But $\left(q_{0}(i A)\right)^{\sim}=q(i A \phi)$ so $i([\Omega(A), q(\phi)])^{\sim} \supseteq q(i A \phi)$.

Therefore $q(i A \phi)^{*} \supseteq-i([\Omega(A), q(\phi)])^{*} \supseteq-i\left[q(\phi)^{*}, \Omega(A)^{*}\right]=-i[q(\phi)$, $\Omega(A)], \quad q(i A \phi)=q(i A \phi)^{*} \supseteq-i([q(\phi), \quad \Omega(A)])^{\sim}=i([\Omega(A), \quad q(\phi)])^{\sim}, q(i A \phi)$ $=i([\Omega(A), q(\phi)])^{2}$.

\section{PART II. Physical interpretation}

1. The elements $f$ of $\mathfrak{F}$, normalized to $\|f\|=1$, will represent states of a many-particle system in such a way that $\left(\left(0 \oplus \cdots \oplus 0 \oplus I^{(n)} \oplus 0 \oplus \cdots\right) f, f\right)$ is the probability that $f$ consists of $n$ particles, where $\left(0 \oplus \cdots \oplus 0 \oplus I^{(n)}\right.$ $\oplus 0 \oplus \cdots)$ is the projection of $\mathfrak{F}$ onto $i \Re^{(n)}$. (See $[3$, p. 637].)

If $f_{n} \in \mathrm{i} \Re^{(n)}$ and $A$ is self-adjoint, then $\left(\Omega(A) f_{n}, f_{n}\right)=((A \otimes I \otimes \cdots \otimes I$ $\left.+\cdots+I \otimes \cdots \otimes I \otimes A) \sim f_{n}, f_{n}\right)$ is the expectation value in the $n$-particle state $f_{n}$, as given in the standard theory, of that $n$-particle observable which corresponds to the single-particle observable $A$. Since $\Omega(A)$ leaves each $i \Re^{(n)}$ invariant, $(\Omega(A) f, f)=\sum_{n=0}^{\infty}\left(\Omega(A) f_{n}, f_{n}\right)$ is the corresponding expectation value in the generalized state $f$ where the total number of particles may not be definitely known.

In particular, if $I$ is the identity operator on $\Re$ and $f \in \mathscr{D}_{\Omega(I)}$, then $\Omega(I)$ $=\sum_{n=0}^{\infty} \oplus n I^{(n)}$ and $(\Omega(I) f, f)=\sum_{n=0}^{\infty} n\left(\left(0 \oplus \cdots \oplus 0 \oplus I^{(n)} \oplus 0 \oplus \cdots\right) f, f\right)$ is the expectation of the total number of particles in the system when the system is in the state $f$.

Similarly, if $P$ is a projection on $\Re$ onto itself corresponding to the property $P$ of the original one-particle system [7, Chap. III, §5], then $(\Omega(P) f, f)$ is the expectation number of particles in the system $\mathfrak{F}$ having the property $P$ since: Let $\left\{\psi_{i}^{(1)}\right\}$ be an orthonormal basis of the subspace $P \Re$ of $\Re$ and $\left\{\psi_{j}^{(0)}\right\}$ an orthonormal basis of its orthogonal complement $(I-P) \Re$. Then elements of the form $i \psi_{k_{1}}^{\left(s_{1}\right)} \otimes \psi_{k_{2}}^{\left(s_{2}\right)} \otimes \cdots \otimes \psi_{k_{n}}^{\left(s_{n}\right)}, s_{i}=0$ or $1, k_{i}=1,2, \cdots ; n=0,1,2, \cdots$, 
form an orthonormal basis of $\mathfrak{F}$ such that $\Omega(P) \mathfrak{i} \psi_{k_{1}}^{\left(s_{1}\right)} \otimes \cdots \otimes \psi_{k_{n}}^{\left(s_{n}\right)}=\mathfrak{i}\left(P \psi_{k_{1}}^{\left(s_{1}\right)}\right)$ $\otimes \psi_{k_{2}}^{\left(s_{2}\right)} \otimes \cdots \otimes \psi_{k_{n}}^{\left(s_{n}\right)}+\mathrm{i} \psi_{k_{1}}^{\left(s_{1}\right)} \otimes\left(P \psi_{k_{2}}^{\left(s_{2}\right)}\right) \otimes \cdots \otimes \psi_{k_{n}}^{\left(s_{n}\right)}+\cdots+\mathrm{i} \psi_{k_{1}}^{\left(s_{1}\right)} \otimes \cdots$ $\otimes\left(P \psi_{k_{n}}^{\left(s_{n}\right)}\right)=\sum_{i=1}^{n}\left(\delta_{s_{i}}^{1} \mathrm{j} \psi_{k_{1}}^{\left(s_{1}\right)} \otimes \cdots \otimes \psi_{k_{n}}^{\left(s_{n}\right)}\right)=m \mathrm{i} \psi_{k_{1}}^{\left(s_{1}\right)} \otimes \cdots \otimes \psi_{k_{n}}^{\left(s_{n}\right)}$, where $\quad m$ $=\sum_{k=1}^{n} \delta_{s_{k}}^{1}=\sum_{k=1}^{n} s_{k}$ is the number of the $\psi_{k_{i}}^{\left(s_{i}\right)}$ which are in $P \Re$. Thus, the eigenvalues of $\Omega(P)$ are the non-negative integers $m$ (occupation numbers of $P \Re)$, and the corresponding eigenvectors $\psi$ such that $\Omega(P) \psi=m \psi$ are those states of $\mathfrak{F}$ representing a system in which exactly $m$ of the particles have the property $P$. For example, if $P=P_{[\phi]}$ (projection on the one-dimensional subspace $[\phi]$ of $\Re$ spanned by $\phi)$, then the eigenvalues of $\Omega\left(P_{[\phi]}\right)$ are the occupation numbers of the state $\phi$ and $\left(\Omega\left(P_{[\phi]}\right) f, f\right)$ is the expectation number of particles having the property that they are in the state $\phi$.

If the above case, since its eigenvectors span the whole Hilbert space $\mathfrak{F}$, it is obvious that $\Omega(P)$ is self-adjoint. Theorem 1 makes the more general statement that if $A$ is an observable, then so is $\Omega(A)$.

Since any self-adjoint $A=\int \lambda d E_{\lambda}$ is approximated by sums of the form $\sum \lambda_{i}\left(E_{\lambda_{i+1}}-E_{\lambda_{i}}\right)$ and $\Omega$ is linear (Theorem 2), $\Omega(A)$ is, in some unspecified sense, approximated by sums of the form $\sum \lambda_{i} \Omega\left(E_{\lambda_{i+1}}-E_{\lambda_{i}}\right)$ where $\Omega\left(E_{\lambda_{i+1}}-E_{\lambda_{i}}\right)$ is that operator which gives the expectation number of particles having the property that their value in $A$ is certainly between $\lambda_{i}$ and $\lambda_{i+1}$, i.e., the eigenvalues of $\Omega\left(E_{\lambda_{i+1}}-E_{\lambda_{i}}\right)$ are occupation numbers of the subspace $\left(E_{\lambda_{i+1}}-E_{\lambda_{i}}\right) \Re$ of $\Re$. We write, symbolically, $\Omega(A) \doteq \int \lambda d \Omega\left(E_{\lambda}\right)$, without trying to indicate the exact type of convergence involved. At any rate, for a dense set of states $f$ we have $(\Omega(A) f, f)=\int \lambda d\left(\Omega\left(E_{\lambda}\right) f, f\right)$ so, very roughly speaking, to find the expectation value of $\Omega(A)$ we take each eigenvalue $\lambda$ of $A$, multiply by the probable number of particles in that eigenstate, and sum over all eigenstates.

The heuristic discussion in the preceding paragraph will not be further rigorized because we can always use $\Omega(A)$ in place of $\lambda \lambda d \Omega\left(E_{\lambda}\right)$. However, in order to show how the mathematical formalism of this paper fits onto a physical theory it will be convenient, at times, to translate that formalism into the conventional terminology of standard works on the subject. For example we can derive the formula $H_{N_{1} N_{2}} \ldots=\sum_{k} \hbar \omega_{k}\left(N_{k}+1 / 2\right)$ of $[10$, p. 34, (6.22)] except that the infinite null-point energy never appears. The single-particle energy operator $H$ is here $H=c\left(m^{2} c^{2}+p^{2}\right)^{1 / 2}$, where $m$ is the mass of the particle, $c$ is the velocity of light, and $p=-i \hbar \nabla=\left(p_{1}, p_{2}, p_{3}\right)$. Now, diagonalizing the maximal-abelian algebra generated by the three operators $p_{1}, p_{2}, p_{3}$, we get $p=\int \lambda d E_{\lambda}$, where the resolution $\left\{E_{\lambda}\right\}$ of the identity consists of the set of all multiplications by characteristic functions defined on the diagonalizing measure space (which is here just momentum space). Hence $H=\int c\left(m^{2} c^{2}+\lambda^{2}\right)^{1 / 2} d E_{\lambda}$, and $\Omega(H) \doteq \int c\left(m^{2} c^{2}+\lambda^{2}\right)^{1 / 2} d \Omega\left(E_{\lambda}\right)$. (We actually have rigorous equality here because the approximating sums on the right converge weakly to $\Omega(H)$ as can be seen from Lebesgue's bounded convergence theorem.) This corresponds exactly to the formula $\sum_{k} \hbar \omega_{k} N_{k}$ (where mo- 
mentum space has been artificially discretified by making ordinary space compact) since $p=\hbar k, \hbar \omega_{k}=c\left(m^{2} c^{2}+p^{2}\right)^{1 / 2}$; and $N_{k}$ gives the number of particles in the momentum eigenstate $k$ just as does $d \Omega\left(E_{\hbar_{k}}\right)$.

The photon case is like that of the scalar mesons, except that the mass $m$ is zero and that we must sum over two polarization states. Again an expression is obtained which is exactly analogous to formula (16.45), p. 117 of [10], without the infinite null-point energy $\sum_{k} \hbar c|k|$.

Similarly, the total momentum $G$ of the field (if by "momentum of the field" we mean the sum of the momenta of the particles associated with the field) is given by $G=\Omega(p) \doteq \int \lambda d \Omega\left(E_{\lambda}\right)$, which corresponds to formula (6.28) of $[10]$ (or (16.45) for photons).

Theorem 2 describes the algebraic properties of $\Omega$. Its extension to Lie algebras of unbounded operators would bring in mathematical complications which can be avoided in most cases by the use of Theorem 1. For example: Theorem 2 shows that the bounded operators $A$ and $B$ commute if and only if their images $\Omega(A)$ and $\Omega(B)$ commute, whereas Theorem 1 (Corollary 1 ) shows that this is also true if $A$ and $B$ are normal. Thus, in the previous discussion, $\boldsymbol{p}$ and $H=c\left(m^{2} c^{2}+p^{2}\right)^{1 / 2}$ commute, so $\boldsymbol{G}=\Omega(\boldsymbol{p})$ and $\Omega(H)$ do also and, as was to have been expected on physical grounds, the total momentum of the field is a constant of its motion. Similarly, since the identity commutes with everything, so does $\Omega(I)$ with every $\Omega(A)$. Physically this means that any observable relating to "particle-like" aspects of the field may be measured with a simultaneous determination of the total number of particles of the field (given by $\Omega(I)$ ). That this is not true of observables relating to "wave-like" aspects of the field will be seen when the field-variables are defined.

If the observable $F(t)$ is a suitably differentiable function of the time $t$, and $H$ is the energy, it is customary to define another observable $\dot{F}(t)$ $=(i / \hbar)[H, F(t)]+(\partial / \partial t) F(t)$ which has the property that $(d / d t)(F(t) \phi(t)$, $\phi(t))=(\dot{F}(t) \phi(t), \phi(t))$. By a purely formal manipulation using Theorem 2 we have $(i / \hbar)[\Omega(H), \Omega(F(t))]+(\partial / \partial t) \Omega(F(t)) \doteq \Omega((i / \hbar)[H, \quad F(t)]+(\partial / \partial t) F(t))$, i.e., $\Omega(F(t)) \doteq \Omega(\dot{F}(t))$; although this formula is true rigorously only if the proper restrictions are placed on $F(t)$ and $H$.

In the above discussions we have several times assumed that $\Omega(H)$ is the total energy of the system. This is permissable when no foreign particles (fields) are present. Interaction forces are ignored and each element of the field moves along its trajectory according to the one-parameter group of unitary operators $\exp (-(i / \hbar) t H)$ exactly as though it were the only particle present. Hence we have by formula (7): $\exp (-(i / \hbar) t \Omega(H))=\sum_{n=0}^{\infty}$ $\oplus(\exp (-(i / \hbar) t H))^{(n)}$.

$H$ is the single-particle Hamiltonian (operating on $\Re$ ). If $A$ operates on $\Re$, let $A_{t}=\exp ((i / \hbar) t H) A \exp (-(i / \hbar) t H)$; if $T$ operates on $\mathfrak{F}$, let $T_{t}$ $=\exp ((i / \hbar) t \Omega(H)) T \exp (-(i / \hbar) t \Omega(H))$. This time-dependence gives us 
the observables in the interaction representation. By Theorem 3 we have $\Omega(A)_{t}=\Omega\left(A_{t}\right)$.

2. The $\omega_{G}(\phi)$ and $\omega_{G}{ }^{*}(\phi)$ of Definition 4 are creation and annihilation operators respectively, though much too generally defined until $G$ is further specified.

Commutation relations involving $\Omega$ and $\omega$ with themselves and with each other are given in Theorems 2, 5, 6, and 8. They will be needed later on in application of the formalism to actual physical situations.

If for $\phi$ in $\Re$ we define a time-dependence by $\phi_{t}=\exp (-(i / \hbar) t H) \phi$, then Theorem 4 says that $\omega_{G}(\phi)_{t}=\omega_{G}\left(\phi_{-t}\right)$ and $\omega_{G}{ }^{*}(\phi)_{t}=\omega_{G}{ }^{*}\left(\phi_{-t}\right)$. More generally let $\exp (i \tau A)$, where $A$ is self-adjoint and $\tau$ is real, be any [8, p. 69] continuous one-parameter group of automorphisms of $\Re$. Then $\mathfrak{F}$, by its construction, goes to $\sum_{n=0}^{\infty} \oplus \exp (i \tau A)^{(n)} \mathfrak{F}$, and an operator $T$ on $\mathfrak{F}$ goes to $T_{\tau}=\sum_{n=0}^{\infty}$ $\bigoplus \exp (i \tau A)^{(n)} T \sum_{n=0}^{\infty} \oplus \exp (-i \tau A)^{(n)}$. For example if $\Re$ is $\Omega_{2}\left(E_{3}\right)$, the set of all square-integrable functions $\phi\left(x_{0}, y_{0}, z_{0}\right)$ on Euclidean 3 -space, then the one-parameter group $\exp \left(i x(1 / i)\left(\partial / \partial x_{0}\right)\right)$ is that generated by translations $\phi_{x}\left(x_{0}, y_{0}, z_{0}\right)=\phi\left(x_{0},-x, y_{0}, z_{0}\right)$ of $E_{3}$.

In connection with such one-parameter groups it is customary to define a derivative by $\left(\partial / \partial_{\tau}\right) B_{\tau}=i\left(\left[B_{\tau}, A\right]\right)^{\sim}$ if $B$ operates on $\Re$. If $T$ operates on $\mathfrak{F}$ then, by (7), the corresponding infinitesimal generator is $i \tau \Omega(A)$, and $(\partial / \partial \tau) T_{\tau}=i\left(\left[T_{\tau}, \Omega(A)\right]\right)^{\sim}$. In particular, by Theorems 4 and 5 , we have $(\partial / \partial \tau) \omega_{G}(\phi)=-\omega_{G}((\partial / \partial \tau) \phi),(\partial / \partial \tau) \omega_{G}{ }^{*}(\phi)=-\omega_{G}{ }^{*}((\partial / \partial \tau) \phi)$, and by Lemma 4

$$
(\partial / \partial \tau) p(\phi)=-p((\partial / \partial \tau) \phi, \quad(\partial / \partial \tau) q(\phi)=-q((\partial / \partial \tau) \phi) .
$$

3. Every observation of a system of $n$ identical particles is invariant under permutation of those particles, i.e., $A=U_{\pi}^{-1} A U_{\pi}$ for the corresponding operator $A$. So all such observables must belong to the centralizer $G_{n}^{\prime}$ of $G_{n}$. The central decomposition of $G_{n}$ decomposes $G_{n}^{\prime}$ into direct factors $P_{\tau} G_{n}^{\prime}$, and reduces $\Re^{(n)}$ to a direct sum of non-interacting subspaces $P_{\tau} \Re^{(n)}$ of wavefunctions which have inequivalent symmetry properties depending on $\tau$. Each $P_{\tau} \Re^{(n)}$ can be further reduced into the direct sum of noninteracting subspaces $P_{\tau, i} \Re^{(n)}, i=1,2, \cdots, m(\tau)$, of wave-functions having equivalent symmetry properties.

Such discussions of the group algebra $G_{n}$ are omittable in view of the present state of physical theory. All particles so far discovered have either obeyed Fermi-Dirac statistics and belonged to the subspace $P_{a_{n}} \Re^{(n)}$, or obeyed Bose-Einstein statistics and belonged to $P_{s_{n}} \Re^{(n)}$. We shall consider the Fermi-Dirac case first.

The superscripts $n_{k}$ in the elements $\left(\phi_{1}^{n_{1}} \cdots \phi_{k}^{n_{k}} \cdots\right)_{A}$ of the orthonormal basis of $\mathfrak{A}$ are occupation numbers, as can be seen from the fact that $\Omega\left(P_{[\phi k]}\right)\left(\phi_{1}^{n_{1}} \phi_{2}^{n_{2}} \cdots\right)_{A}=n_{k}\left(\phi_{1}^{n_{1}} \phi_{2}^{n_{3}} \cdots\right)_{A}$ and $\Omega(I)\left(\phi_{1}^{n_{1}} \phi_{2}^{n_{2}} \cdots\right)_{A}$ $=\left(\sum_{i} n_{i}\right)\left(\phi_{1}^{n_{1}} \phi_{2}^{n_{2}} \cdots\right)_{A}$ where $n_{k}$ equals one or zero according as the state $\phi_{k}$ is occupied or not and $\sum_{i} n_{i}$ is the total number of particles in the system. 
If the $\phi_{m}$ are all eigenvectors of the Hamiltonian, $H \phi_{m}=\lambda_{m} \phi_{m}$, then we get the formulas $\Omega(H) \omega_{A}^{*}\left(\phi_{m}\right)=\omega_{A}^{*}\left(\phi_{m}\right)\left(\Omega(H)-\lambda_{m}\right), \quad \Omega(H) \omega_{A}\left(\phi_{m}\right)=\omega_{A}\left(\phi_{m}\right)(\Omega(H)$ $\left.+\lambda_{m}\right)$ of $[10$, p. 166] by use of Theorem 5 .

When we substitute elements $\phi_{i}$ of $\left\{\phi_{i}\right\}$ into Lemma 1 in place of $\phi$ and $\psi$, we get formula (20.20) on p. 164 of [10]. It follows that if the system is in the state $f \in \mathfrak{A}$, then the expectation number of particles in the state $\phi_{i}$ is $\left\|\omega_{A}^{*}\left(\phi_{i}\right) f\right\|^{2}$, since $\left\|\omega_{A}^{*}\left(\phi_{i}\right) f\right\|^{2}=\left(\omega_{A}\left(\phi_{i}\right) \omega_{A}^{*}\left(\phi_{i}\right) f, \quad f\right)=\left(\Omega\left(\phi_{i} \phi_{i}{ }^{*}\right) f, f\right)$ $=\left(\Omega\left(P_{\left[\phi_{i}\right]}\right) f, f\right)$.

Theorem 6 is a similar generalization of the standard anti-commutation relations $[10$, p. $165,(20.23)]$. The time-dependent anti-commutator brackets are then, by Theorem 4: $\left[\omega_{A}(\phi)_{t}, \omega_{A}^{*}(\psi)_{t^{\prime}}\right]_{+}=\left(\exp \left(\left(\left(t^{\prime}-t\right) / i \hbar\right) H\right) \phi, \psi\right) I$, $\left[\omega_{A}(\phi)_{t}, \omega_{A}(\psi)_{t^{\prime}}\right]_{+}=0,\left[\psi_{A}^{*}(\phi)_{t}, \omega_{A}^{*}(\psi)_{t^{\prime}}\right]_{+}=0$.

$\Omega(A)$ is usually expressed in terms of creation and annihilation operators as $\sum_{i, j} \omega_{G}\left(\phi_{i}\right)\left(A \phi_{j}, \phi_{i}\right) \omega_{G}{ }^{*}\left(\phi_{j}\right)$ (see [3, pp. 634 and 629]). The formal identity of the two expressions in the Fermi-Dirac and Bose-Einstein cases can be seen as follows: Let $A$ be any self-adjoint operator on $\Re$ with pure point spectrum, i.e., such that there exists a set of eigenvectors $\left\{\psi_{i}\right\}, A \psi_{i}=\lambda_{i} \psi_{i}$, which are also an orthonormal basis of $\Re$. Then, using " $\doteq$ " to mean "formally identical," we have $\Omega(A)=\Omega\left(\sum_{i} \lambda_{i} P_{\left[\psi_{i}\right]}\right) \doteq \sum_{i} \lambda_{1} \Omega\left(P_{\left[\psi_{i}\right]}\right)=\sum_{i} \lambda_{i} \omega_{G}\left(\psi_{i}\right) \omega_{G}{ }^{*}\left(\psi_{i}\right)$ by Lemmas 1 or 3 according as $G$ is $A$ or $S$. Therefore $\Omega(A)=\sum_{i}\left(A \psi_{i}, \psi_{i}\right)$ $\omega_{G}\left(\psi_{i}\right) \omega_{G}{ }^{*}\left(\psi_{i}\right)=\sum_{i, j}\left(A \psi_{i}, \psi_{j}\right) \psi_{G}\left(\psi_{j}\right) \omega_{G}^{*}\left(\psi_{i}\right)=\sum_{i, j}\left(A \psi_{i}, \psi_{j}\right) \omega_{G}\left(\sum_{n}\left(\psi_{j}, \phi_{n}\right) \phi_{n}\right)$ $\omega_{G}^{*}\left(\sum_{m}\left(\psi_{i}, \quad \phi_{m}\right) \phi_{m}\right) \doteq \sum_{i, j, m, n}\left(A \psi_{i}, \quad \psi_{j}\right)\left(\psi_{j}, \quad \phi_{n}\right)\left(\left(\psi_{i}, \quad \phi_{m}\right)^{*} \omega_{G}\left(\phi_{n}\right) \omega_{G}{ }^{*}\left(\phi_{m}\right)\right.$ $=\sum_{m, n}\left(A \sum_{i}\left(\phi_{m}, \psi_{i}\right) \psi_{i}, \sum_{j}\left(\phi_{n}, \psi_{j}\right) \psi_{j}\right) \omega_{G}\left(\phi_{n}\right) \omega_{G}{ }^{*}\left(\phi_{m}\right)=\sum_{m, n}\left(A \phi_{m}, \phi_{n}\right)$ $\omega_{G}\left(\phi_{n}\right) \omega_{G}^{*}\left(\phi_{m}\right)$. So $\Omega(A) \doteq \sum_{i, j} \omega_{G}\left(\phi_{i}\right)\left(A \phi_{j}, \phi_{i}\right) \omega_{G}^{*}\left(\phi_{j}\right)$.

Presumably this formula can be extended to hold for any self-adjoint transformation by passing to some kind of limit on both sides, and to an arbitrary transformation by writing it as the sum of its real and imaginary parts and using linearity. We shall not try to justify these manipulations. The form $\Omega(A)$ should be sufficient for all physical applications.

4. A system obeys Bose-Einstein statistics if and only if its wave-function is contained in the subspace $\subseteq$ of symmetric tensors of $\mathfrak{F}$. The superscripts $n_{k}$ in the elements of the orthonormal basis $\left(\phi_{1}^{n_{1}} \phi_{2}^{n_{2}} \ldots\right)_{s}$ of $\mathbb{S}$ are occupation numbers: $\Omega\left(P_{\left[\phi_{k}\right]}\right)\left(\phi_{1}^{n_{1}} \phi_{2}^{n_{2}} \cdots\right)_{s}=n_{k}\left(\phi_{1}^{n_{1}} \phi_{2}^{n_{2}} \cdots\right)_{s}$ so the non-negative integer $n_{k}$ gives the number of particles in the system which are in the state $\phi_{k}$.

Lemma 3 is a formal generalization of formula $(6.18)[10$, p. 33] since: Replace Euclidean space, $E_{3}$, by the 3-dimensional torus; i.e., impose a periodicity condition $[10$, p. 26]. Then momentum space becomes discrete to consist of the orthonormal basis $\left\{\phi_{k}\right\}$ of $\mathfrak{R}_{2}\left(E_{3}\right)$. In the terminology of [10]: $a_{k}=\omega_{s}^{*}\left(\phi_{k}\right), a_{k^{*}}=\omega_{s}\left(\phi_{k}\right)$; and $a_{k} a_{k^{*}}=\omega_{s}^{*}\left(\phi_{k}\right) \omega_{s}\left(\phi_{k}\right)=I+\Omega\left(\phi_{k} \phi_{k^{*}}\right)=1+N_{k}$, and $a_{k^{*}} a_{k}=\omega_{s}\left(\phi_{k}\right) \omega_{s}^{*}\left(\phi_{k}\right)=\Omega\left(\phi_{k} \phi_{k^{*}}\right)=\mathrm{N}_{k}$.

If the system is in the state $f \in \mathbb{S}$, then the expectation number of particles in the state $\phi \in \Re(\|\phi\|=1)$ is $\left\|\omega_{s}^{*}(\phi) f\right\|^{2}$ since $\left\|\omega_{s}^{*}(\phi) f\right\|^{2}=\left(\omega_{s}(\phi) \omega_{s}^{*}(\phi) f, f\right)$ $=\left(\Omega\left(\phi \phi^{*}\right) f, f\right)=\left(\Omega\left(P_{[\phi]}\right) f, f\right)$. 
If $\omega_{s}$ and $\omega_{s}^{*}$ are taken as functions of an orthonormal basis of $\Re$, then Theorem 8 becomes the standard commutation relations for creation and annihilation operators $[10$, p. $34,(6.19)]$. The corresponding time-dependent commutator brackets are $\left[\omega_{s}(\phi)_{t}, \omega_{s}(\psi)_{t^{\prime}}\right]^{\sim}=0,\left[\omega_{s}^{*}(\phi)_{t}, \omega_{s}^{*}(\psi)_{t^{\prime}}\right]^{\sim}=0$, and $\left[\omega_{s}^{*}(\phi)_{t}, \omega_{s}(\psi)_{t^{\prime}}\right]^{\mathcal{N}}=\left(\exp \left(\left(\left(t-t^{\prime}\right) / i \hbar\right) H\right) \psi, \phi\right) I$ as follows from Theorems 4 and 8 . By using these commutation relations it will be possible to carry through the physical theory without having recourse to the singular Dirac $\delta$-function and Jordan-Pauli invariant $D$-function.

The creation and annihilation operators are not normal, so they do not represent observable quantities. However they can, by Definition 6 , be used to construct quantities $p(\phi)$ and $q(\phi)$ which are, by Theorem 10, observables.

It is customary, in the quantum theory of wave fields, to assign to every point $x$ of Euclidean 3-space an operator $q(x)$ representing the value of the field and an operator $p(x)$ representing the corresponding Hamiltonianconjugate momentum at that point. It has been generally recognized, however, that this is an artifice which does not correspond to physical reality (see $[4$, Chap. III, $\S 1 ; 1, \S 2 ; 2]$ ). The only way a field strength can be measured, and therefore the only way a meaning can be given to the concept, is by introducing a test-body into the field. This test-body will always have a finite nonzero volume. It will never be just a point. In other words it will always be an element of a Hilbert space, never an element of Euclidean 3-space; and the field strengths measured will always be averages over a certain volume, never values at a point. Even if the test-body is a single electron and, instead of giving it a nonzero diameter we have assumed the point-model which seems to lie at the basis of quantum mechanics, we are still not allowed to use that point itself in any of our calculations. Only a certain probability distribution appears, again an element of Hilbert space.

In the present formalism, the operators are defined to depend directly on weighted distributions. The second quantization mapping $q$ takes $\Re$ into a set of operators on $\subseteq$; but elements $\phi$ of $\Re$, as opposed to those of $\subseteq$, need not be interpreted as the wave-functions of particles. For example, that field which is felt by a small spherical volume of radius $e^{2} / m c^{2}$ is given by the operator $q(\phi)$ where $\phi$ is a function defined to equal one on the volume in question and zero outside of it. More generally, if $\phi$ is any real squareintegrable function, then it represents that field which is felt by the corresponding mass-distribution; or, alternatively, it can be interpreted as a probability-distribution and used in calculating expectation values. Squareintegrability, the condition that puts $\phi$ in $\Re$, is not necessary, but is very convenient and sufficiently weak to include the physically interesting cases.

The distribution may be defined, not only on Euclidean 3-space, but on any measure space $X$ such that $\Re=\Omega_{2}(X)$. Thus Fourier transforms are automatically taken care of and momentum space is handled exactly as is ordinary space. 
If the $\phi$ and $\psi$ of Theorem 11 are elements of an orthonormal basis $\left\{\phi_{i}\right\}$ of $\Re$, then $\left(\left[q\left(\phi_{i}\right), p\left(\phi_{j}\right)\right]\right)^{\sim}=i \delta_{i}^{j}$ and $\left(\left[q\left(\phi_{i}\right), q\left(\phi_{j}\right)\right]\right)^{\sim}=\left(\left[p\left(\phi_{i}\right), p\left(\phi_{j}\right)\right]\right)^{\sim}=0$; which are the standard commutation relations $[10$, p. 27, (5.4)]. Corresponding commutation relations for the time-dependent operators $[10$, p. 20 , (4.12)] are, by Theorems 4 and 11:

$$
\begin{aligned}
{\left[\left(q(\phi)_{t}, q(\psi)_{t^{\prime}}\right]\right)^{\sim}=} & (1 / 2)\left(\left(\exp \left(\left(\left(t-t^{\prime}\right) / i \hbar\right) H\right) \psi, \phi\right)\right. \\
& \left.-\left(\exp \left(\left(\left(t^{\prime}-t\right) / i \hbar\right) H\right) \phi, \psi\right)\right) I, \\
\left(\left[q(\phi)_{t}, p(\psi)_{t^{\prime}}\right]\right)^{\sim}= & (i / 2)\left(\left(\exp \left(\left(\left(t-t^{\prime}\right) / i \hbar\right) H\right) \psi, \phi\right)\right. \\
& \left.+\left(\exp \left(\left(\left(t^{\prime}-t\right) / i \hbar\right) H\right) \phi, \psi\right)\right) I, \\
\left(\left[p(\phi)_{t}, p(\psi)_{t^{\prime}}\right]\right) \sim= & (1 / 2)\left(\left(\exp \left(\left(\left(t-t^{\prime}\right) / i \hbar\right) H\right) \psi, \phi\right)\right. \\
& \left.-\left(\exp \left(\left(\left(t^{\prime}-t\right) / i \hbar\right) H\right) \phi, \psi\right)\right) I .
\end{aligned}
$$

If we wish to examine the effects of the field taken over two bounded subsets $S$ and $S^{\prime}$ of Euclidean 3-space, situated at times $t$ and $t^{\prime}$ respectively, then we use the time-independent characteristic functions $\phi=\chi_{s}$ and $\psi=\chi_{s^{\prime}}$. If $S$ and $S^{\prime}$ are outside of each other's light-cones, then $\left(\exp \left(\left(\left(t-t^{\prime}\right) / i \hbar\right) H\right) \psi, \phi\right)$ $=0$ since $H$ must be constructed so as to prohibit exp $((t / i \hbar) H)$ from propagating a disturbance faster than light and therefore $\exp \left(\left(\left(t-t^{\prime}\right) / i \hbar\right) H\right) \psi$, considered as a function on Euclidean 3-space, is zero on the set $S$. Therefore, by (11), the operators commute and we get the usual result that measurements of the field quantities may be taken over mutually space-like regions of space-time without uncertainty restrictions. (See [1].)

Although these results may seem, at first glance, to have been obtained by methods rather different from those normally employed, this is not the case. There is a complete analogy which can be verified at each step, and the Jordan-Pauli $D$-function can, if we are willing to accept the customary relaxation of rigor, be derived directly from (11).

Rather than discuss this analogy more thoroughly, it will be of greater interest to take the following actual application from meson theory and work it through using the commutation relations and the general mathematical structure which has been developed in the preceding pages.

Ascalar meson obeys the positive-definite Hamiltonian $H=c\left(m^{2} c^{2}+\hbar^{2} k^{2}\right)^{1 / 2}$, where $m$ is its mass and $k=-i \nabla$. (See $[10, \S 6]$.) Therefore the energy of the meson field is given by $\Omega(H)$. Now suppose that a foreign mass-distribution, represented by the real square-integrable function $\rho$, is introduced into the field; and that this fixed mass-distribution has the property that it can react with the field by absorbing and emitting mesons from and into it. Then an interaction term must be added to $\Omega(H)$ in order properly to describe this altered system. Let $\tilde{p}(\phi)=p\left(H^{1 / 2} \phi\right)$ and $\tilde{q}(\phi)=q\left(H^{-1 / 2} \phi\right)[10$, p. 34, (6.20)].

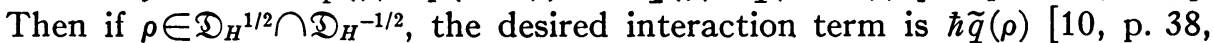
(7.5) ], and the total energy of the field is $W=\Omega(H)+\hbar \tilde{q}(\rho)$. In order to study 
this expression, define a unitary transformation $U=\exp \left(i \hbar \tilde{p}\left(H^{-2} \rho\right)\right)$ of $\mathfrak{S}$.

Now there is some computing to be done which would be tedious to tackle in full rigor; so, this being just an illustration of the theory, not a part of it, we will again use the symbol $\doteq$ to mean formal equality and will use the identity

$$
[\exp (A), B] \doteq([A, B]+(1 / 2)[A,[A, B]]) \exp (A)
$$

which is formally true for any $A, B$ such that $[A,[A,[A, B]]] \doteq 0$. Then $\left[-i \hbar \tilde{p}\left(H^{-2} \rho\right), \hbar \tilde{q}(\rho)\right] \doteq-\hbar^{2}\left\|H^{-1} \rho\right\|^{2} I$ (by Theorem 11 ), so [U $\left.U^{-1}, \hbar \tilde{q}(\rho)\right]$ $\doteq-\hbar^{2}\left\|H^{-1} \rho\right\|^{2} U^{-1}$ (by (12)), and $U^{-1} \hbar \tilde{q}(\rho) U \doteq \hbar \tilde{q}(\rho)-\hbar^{2}\left\|H^{-1} \rho\right\|^{2} I$. Also

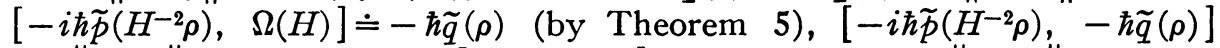
$\doteq \hbar^{2}\left\|H^{-1} \rho\right\|^{2} I$ (as above); so $\left[U^{-1}, \Omega(H)\right] \doteq\left(-\hbar \tilde{q}(\rho)+\left(\hbar^{2} / 2\right)\left\|H^{-1} \rho\right\|^{2}\right) U^{-1}$ (by (12)), and $U^{-1} \Omega(H) U \doteq \Omega(H)-\hbar \tilde{q}(\rho)+\left(\hbar^{2} / 2\right)\left\|H^{-1} \rho\right\|^{2} I$. Therefore $U^{-1} W U$ $\doteq \Omega(H)-\left(\hbar^{2} / 2\right)\left\|H^{-1} \rho\right\|^{2} I$.

Since $U^{-1} W U$ has the same spectrum as $W$, this means that the entire effect of the foreign distribution $\rho$ has been to lower the energy of the system by an amount $\left(\hbar^{2} / 2\right)\left\|H^{-1} \rho\right\|^{2}$.

$H$ is diagonal on momentum space, so $\left(\hbar^{2} / 2\right)\left\|H^{-1} \rho\right\|^{2}=\left(\hbar^{2} / 2\right)\left(H^{-2} \rho, \rho\right)$ $=\left(\hbar^{2} / 2\right) \iiint\left(\rho \dagger(k) \rho \dagger(k)^{*} / c^{2}\left(m^{2} c^{2}+\hbar^{2} k^{2}\right)\right) d k_{1} d k_{2} d k_{3}$, where $\rho \dagger(k)$ is the Fourier transform of $\rho(\mathbf{x})$. Let $V(\boldsymbol{x})=(1 / 2 \pi)^{3} \iiint-\exp (i \boldsymbol{k} \cdot \mathbf{x}) / c^{2}\left(m^{2} c^{2} \hbar^{-2}+k^{2}\right) d k_{1} d k_{2} d k_{3}$ $=-\exp (-m c|x| / \hbar) / 4 \pi c^{2}|\mathrm{x}|\left[10\right.$, p. 43-44, (7.14-15)]. Then $-\hbar^{2} / c^{2}\left(m^{2} c^{2}\right.$ $\left.+\hbar^{2} k^{2}\right)$ is the Fourier transform $\widehat{V}(k)$ of $V(x)$, so we can write $\left(\hbar^{2} / 2\right)\left\|H^{-1} \rho\right\|^{2}$ $=-(1 / 2) \iiint \widehat{V}(\boldsymbol{k}) \rho \dagger(\boldsymbol{k}) \rho \dagger(\boldsymbol{k})^{*} d k_{1} d k_{2} d k_{3}$. But multiplication on momentum space becomes convolution on ordinary space, so $\left(\hbar^{2} / 2\right)\left\|H^{-1} \rho\right\|^{2}$ $=-(1 / 2) \iiint(V * \boldsymbol{\rho})^{\sim} \dagger(\boldsymbol{k}) \rho \dagger(\boldsymbol{k})^{*} d k_{1} d k_{2} d k_{3}$. Finally, the Fourier transform is a unitary operation so $\left(\hbar^{2} / 2\right)\left\|H^{-1} \rho\right\|^{2}=-(1 / 2) \iiint\left(V^{*} \rho\right)(\boldsymbol{x}) \rho(\boldsymbol{x})^{*} d x_{1} d x_{2} d x_{3}$ and the entire expression can be written $U^{-1} W U=\Omega(H)+(1 / 2)$ $\iint V(\mathrm{x}-\mathrm{y}) \rho(\mathrm{x}) \rho(\mathrm{y}) d^{3} \mathbf{x} d^{3} \mathrm{y} I$.

Thus, the interaction energy appears as a screened Coulomb potential $V(\mathbf{x})=-\exp (-m c|\mathbf{x}| / \hbar) / 4 \pi c^{2}|\mathbf{x}|$ of the mass-distribution $\rho$ with respect to itself. The meson field acts as an attracting force which the nucleons exert on each other.

This derivation is analogous to the one originally given by Yukawa, yet that which in Yukawa's treatment corresponds to our $\Omega(H)$ is really quite another thing. The fact that both methods will agree rests essentially on the following formal coincidence.

Assume that all elements of the orthonormal basis $\left\{\phi_{i}\right\}$ of $\Re$ are eigenvectors of the Hamiltonian $H, H \phi_{i}=\lambda_{i} \phi_{i}, \lambda_{i}>0$. Then, since the energy of a classical harmonic oscillator is $(1 / 2)\left(p^{2}+\lambda^{2} q^{2}\right)$, write $\sum_{i}(1 / 2)\left(\widetilde{p}\left(\phi_{i}\right)^{2}\right.$ $\left.+\lambda_{i}^{2} \tilde{q}\left(\phi_{i}\right)^{2}\right)$ for the total energy of the field [10, p. 33, (6.14)] instead of $\Omega(H)$. Then, manipulating this expression in a purely formal fashion, we get $\sum_{i} \quad(1 / 2)\left(\tilde{p}\left(\phi_{i}\right)^{2}+\lambda_{i}^{2} q\left(\phi_{i}\right)^{2}\right)=\sum_{i} \quad(1 / 2)\left(p\left(\lambda_{i}^{1 / 2} \phi_{i}\right)^{2}+\lambda_{i}^{2} q\left(\left(\lambda_{i}^{-1}\right)^{1 / 2} \phi_{i}\right)^{2}\right)$ $=\sum_{i}\left(\lambda_{i} / 2\right)\left(p\left(\phi_{i}\right)^{2}+q\left(\phi_{i}\right)^{2}\right) \doteq \sum_{i}\left(\lambda_{i} / 2\right)\left(\left(i / 2^{1 / 2}\right)\left(\omega_{s}\left(\phi_{i}\right)-\omega_{s}^{*}\left(\phi_{i}\right)\right)\right)^{2} \pm\left(\left(1 / 2^{1 / 2}\right)\right.$ 
$\left.\left.\left(\omega_{s}\left(\phi_{i}\right)+\omega_{s}^{*}\left(\phi_{i}\right)\right)\right)^{2}\right)=\sum_{i} \quad\left(\lambda_{i} / 4\right)\left(-\left(\omega_{s}\left(\phi_{i}\right)-\omega_{s}^{*}\left(\phi_{i}\right)\right)^{2}+\left(\omega_{s}\left(\phi_{i}\right)+\omega_{s}^{*}\left(\phi_{i}\right)\right)^{2}\right)$ $\doteq \sum_{i}\left(\lambda_{i} / 4\right)\left(2 \omega_{s}\left(\phi_{i}\right) \omega_{s}^{*}\left(\phi_{i}\right)+2 \omega_{s}^{*}\left(\phi_{i}\right) \omega_{s}\left(\phi_{i}\right)\right)=\sum_{i}\left(\lambda_{i} / 4\right)\left(4 \Omega\left(\phi_{i} \phi_{i}^{*}\right)+2\left(\phi_{i}, \phi_{i}\right) I\right)$ (by Lemma 3) $=\sum_{i} \lambda_{i}\left(\Omega\left(P_{\left[\phi_{i}\right]}\right)+(1 / 2) I\right)=\sum_{i} \Omega\left(\lambda_{i} P_{\left[\phi_{i}\right]}\right)+\sum_{i}\left(\lambda_{i} / 2\right) I$ $=\Omega\left(\sum_{i} \lambda_{i} P_{\left[\phi_{i}\right]}\right)+\sum_{i}\left(\lambda_{i} / 2\right) I=\Omega(H)+\sum_{i}\left(\lambda_{i} / 2\right) I$.

The highly divergent $\sum_{i}\left(\lambda_{i} / 2\right)$ is the well known infinite null-point energy. Ignoring this infinite term, as is customary, we get $\Omega(H)$ $\doteq \sum_{i}(1 / 2)\left(\tilde{p}\left(\phi_{i}\right)^{2}+\lambda_{i}^{2} q\left(\phi_{i}\right)^{2}\right)$ (an expression not needed in the present formalism).

That relativistic invariance can be a built-in feature of the theory is illustrated by the following treatment of the electromagnetic field.

Before discussing the field itself it will be necessary to describe the invariant wave-equation obeyed by a single photon. Like the Dirac electron, the photon will be a four-component vector function $\phi(x, y, z)=\left(\phi_{1}(x, y, z)\right.$, $\left.\phi_{2}(x, y, z), \phi_{3}(x, y, z), \phi_{4}(x, y, z)\right)$ defined on Euclidean 3-space $E_{3} . \Re$ will be the set of all such complex-valued vector functions $\psi$ which are squareintegrable: $\psi=\phi_{1} \oplus \phi_{2} \oplus \phi_{3} \oplus \phi_{4}$ with $\phi_{i}$ in $\Omega_{2}\left(E_{3}\right)$. However, the Dirac wavefunction is a spinor transforming as a (1/2)-integral representation of the Lorentz group, whereas the four components of the photon wave-function will transform directly under the Lorentz group itself. In order to express this fact we write $\Re$ as the tensor product $\Re=\Omega_{2}\left(E_{3}\right) \otimes \Omega_{4}$, where $\Re_{4}$ is a fourdimensional Hilbert space; then if $\rho_{1}, \rho_{2}, \rho_{3}, \rho_{4}$ is an orthonormal basis of $\Omega_{4}$, any $\psi$ in $\Omega_{2}\left(E_{3}\right) \otimes \Omega_{4}$ can be written uniquely as $\psi=\phi_{1} \otimes \rho_{1}+\phi_{2} \otimes \rho_{2}+\phi_{3} \otimes \rho_{3}$ $+\phi_{4} \otimes \rho_{4}$, giving us the isomorphism with $\phi_{1} \oplus \phi_{2} \oplus \phi_{3} \oplus \phi_{4}$ in the previous representation $\Re=\Omega_{2}\left(E_{3}\right) \oplus \Omega_{2}\left(E_{3}\right) \oplus \Omega_{2}\left(E_{3}\right) \oplus \Omega_{2}\left(E_{3}\right)$. Time-dependence is given by the Hamiltonian $H=c k \otimes I$, where $k=\left(k_{x}^{2}+k_{y}^{2}+k_{z}^{2}\right)^{1 / 2}, \quad k_{x}=-i \hbar(\partial / \partial x), k_{y}$ $=-i \hbar(\partial / \partial y), k_{z}=-i \hbar(\partial / \partial z)$. Now we can describe the action of the Lorentz group on $\Re=\Omega_{2}\left(E_{3}\right) \otimes \Omega_{4}$ by stating that it transforms the basis elements $\rho_{1}, \rho_{2}, \rho_{3}, \rho_{4}$ of $\Omega_{4}$ contragrediently to $x, y, z, c t$, and that it transforms the time-dependent element $\exp (-(i c t / \hbar) k) \phi$ of $R_{2}\left(E_{3}\right)$ as a scalar function of space-time, i.e., if $x, y, z, c t$ goes to $x^{\prime}, y^{\prime}, z^{\prime}, c t^{\prime}$ under the Lorentz transformation, then $\phi^{\prime}\left(x^{\prime}, y^{\prime}, z^{\prime}, c t^{\prime}\right)=\phi(x, y, z, c t)$. The equation of motion $(1 / c)(\partial / \partial t) \phi(x, y, z, c t)=\left(\left(\partial^{2} / \partial x^{2}\right)+\left(\partial^{2} / \partial y^{2}\right)+\left(\partial^{2} / \partial z^{2}\right)\right)^{1 / 2} \phi(x, y, z, c t)$ is obviously Lorentz-invariant. (Inversions of the time-axis are excluded.)

In order to show how $\Omega_{4}$ determines polarization states, we write it as $\Omega_{4}=\Omega_{2} \otimes \Omega_{2}{ }^{*}$ where $\Omega_{2}$ and $\Omega_{2}{ }^{*}$ are two-dimensional Hilbert spaces. The simply-connected covering group of the proper Lorentz group is the set $S L(2)$ of all unimodular linear transformations on spin-space $\Omega_{2}$ (see $[9, \S 20$, p. 78]). Let $\eta_{1}, \eta_{2}$ be an orthonormal basis of $\Omega_{2}$, and $\eta_{1}{ }^{*}, \eta_{2}{ }^{*}$ one of $\Omega_{2}{ }^{*}$; then $\eta_{1} \otimes \eta_{2}{ }^{*}$, $\eta_{2} \otimes \eta_{1}{ }^{*}, \eta_{1} \otimes \eta_{1}{ }^{*}, \eta_{2} \otimes \eta_{2}{ }^{*}$ is an orthonormal basis of $\Omega_{2} \otimes \Omega_{2}{ }^{*}$ and any element $\rho$ of $\Omega_{4}$ can be written $\rho=c_{11} \eta_{1} \otimes \eta_{1}{ }^{*}+c_{12} \eta_{1} \otimes \eta_{2}{ }^{*}+c_{21} \eta_{2} \otimes \eta_{1}{ }^{*}+c_{22} \eta_{2} \otimes \eta_{2}{ }^{*}$ (as in $\left[9\right.$, p. 79, (20.4) ]). Then $\rho_{1}=\left(1 / 2^{1 / 2}\right)\left(\eta_{1} \otimes \eta_{2}{ }^{*}+\eta_{2} \otimes \eta_{1}{ }^{*}\right), \rho_{2}=-\left(i / 2^{1 / 2}\right)\left(\eta_{1} \otimes \eta_{2}{ }^{*}\right.$ $\left.-\eta_{2} \otimes \eta_{1}^{*}\right), \quad \rho_{3}=\left(1 / 2^{1 / 2}\right)\left(\eta_{1} \otimes \eta_{1}^{*}-\eta_{2} \otimes \eta_{2}^{*}\right)^{-}, \quad \rho_{4}=\left(1 / 2^{1 / 2}\right)\left(\eta_{1} \otimes \eta_{1}^{*}+\eta_{2} \otimes \eta_{2}^{*}\right)$ is also an orthonormal basis of $\Omega_{4}$. 
Every proper Lorentz transformation $\theta$ is represented by some transformation $a(\theta)$ in $S L(2)$. (This representation is two-valued, but it makes no difference which value we choose.) Let $\bar{a}(\theta)$ be the transformation on $\Omega_{2}{ }^{*}$ such that the elements of its matrix with respect to the basis $\eta_{1}{ }^{*}, \eta_{2}{ }^{*}$ are the complex-conjugates of those of $a(\theta)$ with respect to $\eta_{1}, \eta_{2}$. Then the mapping $\theta \rightarrow a(\theta) \otimes \bar{a}(\theta)$ is (by $\left[9\right.$, p. 78]) our original representation on $\Omega_{4}:(a(\theta)$ $\otimes \bar{a}(\theta)\left(x_{1} \rho_{1}+x_{2} \rho_{2}+x_{3} \rho_{3}+x_{4} \rho_{4}\right)=x_{1}^{\prime} \rho_{1}+x_{2}^{\prime} \rho_{2}+x_{3}^{\prime} \rho_{3}+x_{4}^{\prime} \rho_{4}$ where $x_{1}^{2}+x_{2}^{2}+x_{3}^{2}-x_{4}^{2}$ $=x_{1}^{\prime 2}+x_{2}^{\prime 2}+x_{3}^{\prime 2}-x_{4}^{\prime 2}$. The spin-matrices

$$
\sigma_{x}=\left(\begin{array}{ll}
0 & 1 \\
1 & 0
\end{array}\right), \sigma_{y}=\left(\begin{array}{rr}
0 & -i \\
i & 0
\end{array}\right), \sigma_{z}=\left(\begin{array}{rr}
1 & 0 \\
0 & -1
\end{array}\right)
$$

correspond to infinitesimal rotations of $E_{3}$ about the $x, y$, and $z$ axes respectively. The $\rho_{i}$ are eigenvectors of these infinitesimal rotations:

$$
\begin{array}{lll}
\sigma_{x} \otimes \bar{\sigma}_{x} \rho_{1}=+\rho_{1}, & \sigma_{y} \otimes \bar{\sigma}_{y} \rho_{1}=-\rho_{1}, & \sigma_{z} \otimes \bar{\sigma}_{z} \rho_{1}=-\rho_{1}, \\
\sigma_{x} \otimes \bar{\sigma}_{x} \rho_{2}=-\rho_{2}, & \sigma_{y} \otimes \bar{\sigma}_{y} \rho_{2}=+\rho_{2}, & \sigma_{z} \otimes \bar{\sigma}_{z} \rho_{2}=-\rho_{2}, \\
\sigma_{x} \otimes \bar{\sigma}_{x} \rho_{3}=-\rho_{3}, & \sigma_{y} \otimes \bar{\sigma}_{y} \rho_{3}=-\rho_{3}, & \sigma_{z} \otimes \bar{\sigma}_{z} \rho_{3}=+\rho_{3}, \\
\sigma_{x} \otimes \bar{\sigma}_{x} \rho_{4}=+\rho_{4}, & \sigma_{y} \otimes \bar{\sigma}_{y} \rho_{4}=+\rho_{4}, & \sigma_{z} \otimes \bar{\sigma}_{z} \rho_{4}=+\rho_{4} .
\end{array}
$$

Therefore, a photon $\psi$ traveling in the $z$-direction and polarized in the $x$-direction (i.e., $\psi=\phi \otimes \rho_{1}$ where $\phi$, as a function on momentum space, is zero outside of a small volume about the positive $z$-axis) belongs to the eigenvalue +1 of $I \otimes\left(\sigma_{x} \otimes \bar{\sigma}_{x}\right)$ and -1 of $I \otimes\left(\sigma_{y} \otimes \bar{\sigma}_{y}\right)$ just as expected on physical grounds. There are two more polarization states still unaccounted for but these will be automatically eliminated by the requirement of relativistic invariance and a restriction of the wave-functions to a certain subspace $\mathfrak{B}$ of $\Re$ later required by the Lorentz condition $(\partial / \partial x) A_{x}+(\partial / \partial y) A_{y}+\left(\partial / \partial_{z}\right) A_{z}$ $+(1 / c)(\partial / \partial t) \Phi=0$ on the as yet undefined four-potential field operators $A_{x}, A_{y}, A_{z}, \Phi$.

The inner product on $\Re=\Omega_{2}\left(E_{3}\right) \otimes \Omega_{4}$ is invariant under rotations of $E_{3}$ (since they correspond to the unitary transformations in $S L(2)$ ) but is not invariant under the Lorentz group. To form expectation values we need an operator $\mathfrak{g}^{\prime}$ on $\Re_{4}$ defined by $\mathfrak{g}^{\prime} \rho_{i}=\rho_{i}$ for $i=1,2,3, \mathfrak{g}^{\prime} \rho_{4}=-\rho_{4}$. Then $I \otimes \mathfrak{g}^{\prime}$ maps a covariant element $\psi \dagger=\phi_{1} \oplus \phi_{2} \oplus \phi_{3} \oplus \phi_{4}$ of $\Re$ (i.e., $\psi \dagger$ transforms contragrediently to $x, y, z, c t$ under the Lorentz group) into a contragredient element $\psi=I \otimes g^{\prime} \psi \dagger=\phi_{1} \oplus \phi_{2} \oplus \phi_{3} \oplus-\phi_{4}$. (We shall always denote covariant elements with the dagger and contravariant elements without.) $\mathfrak{g}^{\prime}$ is obviously. relativistically invariant. Explicity it can be written $\mathfrak{g}^{\prime}=(1 / 2)\left(I \otimes I-\sigma_{x} \otimes \bar{\sigma}_{x}\right.$ $-\sigma_{y} \otimes \bar{\sigma}_{y}-\sigma_{z} \otimes \bar{\sigma}_{z}$ ) (by (13)) and invariance is once more easily recognized from the fact that $\sigma_{x}, \sigma_{y}, \sigma_{z}, I$ transform like the covariant components of a world-vector.

In order to get the subspace $\mathfrak{P} \dagger$ of all those covariant wave-functions in $\Re$ which represent photons, we need to impose on $\psi \dagger=\phi_{1} \oplus \phi_{2} \oplus \phi_{3} \oplus \phi_{4}$ the 
conditions $(\partial / \partial x) \phi_{1}+(\partial / \partial y) \phi_{2}+(\partial / \partial z) \phi_{3}-(1 / c)(\partial / \partial t) \phi_{4}=0$. Since $((\partial / \partial x)$, $(\partial / \partial y),(\partial / \partial z),-(1 / c)(\partial / \partial t))$ is contravariant and $\left(\phi_{1}, \phi_{2}, \phi_{3}, \phi_{4}\right)$ is covariant, this condition is invariant. On momentum space it is equivalent to

$$
k_{x} \phi_{1}+k_{y} \phi_{2}+k_{z} \phi_{3}+k \phi_{4}=0 .
$$

If $\psi$ obeys (14) at time $t=0$, it will obey it for all times since $\exp (-(i / \hbar) t H) \psi \dagger=\exp (-(i / \hbar) t c k) \phi_{1} \oplus \exp (-(i / \hbar) t c k) \phi_{2} \oplus \exp (-(i / \hbar) t c k) \phi_{3}$ $\oplus \exp (-(i / \hbar) t c k) \phi_{4} \cdot \mathfrak{B} \dagger$, the set of all such $\psi \dagger$, is a closed linear manifold in $\Re$ since it is the set of all square-integrable vector functions $\left(\phi_{1}(k), \phi_{2}(k)\right.$, $\left.\phi_{3}(k), \phi_{4}(k)\right)$ perpendicular at each point $k$ of momentum space to the given vector $\left(k_{x}, k_{y}, k_{z}, k\right)$ also depending on $k$.

To every photon is assigned a contravariant wave-function $\psi$ and a covariant wave-function $\psi \dagger . I \otimes g^{\prime}$ is a self-adjoint, unitary transformation of $\Re$ which is an isomorphism of the subspace $\mathfrak{B}$ of all contravariant wave-functions with the subspace $\mathfrak{P} \dagger$ of all covariant wave-functions. It permutes corresponding elements: $I \otimes \mathfrak{g}^{\prime} \psi=\psi \dagger, I \otimes \mathfrak{g}^{\prime} \psi \dagger=\psi$.

If $A$ is any normal operator on $\Re$, we define its expectation value in the state $\psi$ to be $(A \psi, \psi \dagger)$. This value is real for all $\psi$ if and only if $A=A \dagger$, where $A \dagger=\left(I \otimes \mathfrak{g}^{\prime}\right) A^{*}\left(I \otimes \mathfrak{g}^{\prime}\right)$, as follows from $(A \psi, \psi \dagger)=(\psi, \quad(A \dagger \psi) \dagger)$ $=(A \dagger \psi, \psi \dagger)^{*}$. So we define the observables in this theory to be those operators $A$ such that $A=A \dagger$. In particular, any operator on $\Re$ of the form $A=B \otimes I$, where $B$ is a self-adjoint operator on $\mathfrak{R}_{2}\left(E_{3}\right)$, is an observable: $B \otimes I=(B \otimes I) \dagger$. For example, the probability that a photon will be found in a region $D$ of Euclidean 3-space is given by $P_{D} \otimes I$, where $P_{D}$ is that projection operating on $\Omega_{2}\left(E_{3}\right)$ as multiplication by the characteristic function of $D$.

Any photon $\psi$ can be written $\psi=\psi_{H}+\psi_{V}+\psi_{k}+\psi_{c t}$ in such a way that the four components $\psi_{H}, \psi_{V}, \psi_{k}, \psi_{c t}$ are, as vector-valued functions on momentum space, at every point orthogonal to each other; $\psi_{H}$ and $\psi_{V}$ are orthogonal at $k$ to $k$ and represent horizontal and vertical polarization, $\psi_{k}$ is parallel to $k$ and represents longitudinal polarization, and $\psi_{c t}$ is orthogonal to 3 -space and represents scalar polarization. But the condition (14) (applied to contravariant elements) means $0 \cdot \phi_{H}(k)+0 \cdot \phi_{V}(k)+k \phi_{k}(k)-k \phi_{c t}(k)=0$ for every $k$, where the $\phi_{r}$ for $r=H, V, k, c t$ are complex-valued functions such that $\psi_{r}(k)=e_{r}(k) \phi_{r}(k)$, the $e_{r}(k)$ being the new orthonormal basis at $k$. Therefore $\phi_{k}(k)=\phi_{c t}(k)$. Consequently the longitudinal and scalar components of the photon tend to cancel each other out of expectation values. For example, the energy of $\psi$ is given by $(H \psi, \psi \dagger)=\left(c k \otimes I \phi_{1} \oplus \phi_{2} \oplus \phi_{3} \oplus \phi_{4}\right.$, $\left.\phi_{1} \oplus \phi_{2} \oplus \phi_{3} \oplus-\phi_{4}\right)=c\left(k \phi_{1} \oplus k \phi_{2} \oplus k \phi_{3} \oplus k \phi_{4}, \quad \phi_{1} \oplus \phi_{2} \oplus \phi_{3} \oplus-\phi_{4}\right)=c\left(k \phi_{H}, \quad \phi_{H}\right)$ $+c\left(k \phi_{V}, \phi_{V}\right)+c\left(k \phi_{k}, \phi_{k}\right)-c\left(k \phi_{c t}, \phi_{c t}\right)=c\left(k \phi_{H}, \phi_{H}\right)+c\left(k \phi_{V}, \phi_{V}\right)=\left(H\left(\psi_{H}+\psi_{V}\right)\right.$, $\left.\left(\psi_{H}+\psi_{V}\right) \dagger\right)$. The longitudinal and scalar components make no contribution. The same is true of momentum and any function of the momentum operators. Only the horizontal and vertical polarization states remain, and these have the desired spin properties as already noted. 
Formation of expectation values by $(A \psi, \psi \dagger)$ instead of by $(A \psi, \psi)$ does not change the fundamental postulates or methods of quantum mechanics as given in [7]. In any fixed Lorentz frame this new method of forming expectation values can be reformulated so as to be equivalent to the old one. Only in passing from one frame to another does the full mechanism of the new method come into play in a manner which is not equivalent to the old.

Now that the single-photon theory has been taken care of, we can apply the previously discussed method of second-quantization to obtain an electromagnetic field theory.

As the relativistically invariant metric on $\subseteq$ we take the self-adjoint unitary transformation $\mathfrak{g}=\sum_{n=0}^{\infty} \oplus\left(I \otimes \mathfrak{g}^{\prime}\right)^{(n)}$ ( $\mathfrak{g}$ for S. N. Gupta, whose paper Theory of longitudinal photons in quantum electrodynamics, Proceedings of the Physical Society, vol. A63 (1950), p. 681, suggested this indefinite metric and the consequent elimination of longitudinal and scalar photons). The expectation value of any observable $T$ on $\mathfrak{S}$, in the state $f$, is given by $(T f, f \dagger)$ where $f \dagger=\mathrm{gf}$. If $T \dagger=\mathfrak{g} T^{*} \mathrm{~g}$, then we require $T \dagger=T$ as before. In particular, any operator of the form $\Omega(A)$ with $A \dagger=A$ is an observable since $(\Omega(A)) \dagger$ $=\Omega(A \dagger)$ by $(7)$ and Theorems 3 and 1 . Longitudinal and scalar polarization states again cancel each other out as above.

We must redefine the field operators $p$ and $q$ in the obvious way: $P(\psi)$ $=\left(i / 2^{1 / 2}\right)\left(\left[\omega_{s}(\psi)-\omega_{s}^{*}(\psi \dagger)\right]\right)^{\sim}, Q(\psi)=\left(1 / 2^{1 / 2}\right)\left(\left[\omega_{s}(\psi)+\omega_{s}^{*}(\psi \dagger)\right]\right)^{\sim}, \quad \psi \in \Re$, in order to get relativistic invariance and real expectation values. The indicated closures exist because $[8$, p. 30] the adjoints are densely defined. The commutation relations $\left(\left[Q(\psi), Q\left(\psi^{\prime}\right)\right]\right)^{1 / 2}=(1 / 2)\left(\left(\psi^{\prime}, \psi \dagger\right)-\left(\psi, \psi^{\prime} \dagger\right)\right) I,([Q(\psi)$, $\left.\left.P\left(\psi^{\prime}\right)\right]\right)^{\sim}=(i / 2)\left(\left(\psi^{\prime}, \quad \psi \dagger\right)+\left(\psi, \psi^{\prime} \dagger\right)\right) I, \quad\left(\left[P(\psi), \quad P\left(\psi^{\prime}\right)\right]\right)^{\sim}=(1 / 2)\left(\left(\psi^{\prime}, \psi \psi\right)\right.$ $\left.-\left(\psi, \psi^{\prime} \dagger\right)\right) I$ are proven exactly as in Theorem 11 , and are unchanged except that the standard inner product $(f \cdot g)$ is replaced by the relativistic one $(f \cdot g \dagger)$.

Because of the relations $Q\left(\phi \otimes \rho_{i}\right)=q\left(\phi \otimes \rho_{i}\right), i=1,2,3, Q\left(\phi \otimes \rho_{4}\right)$ $=-i p\left(\phi \otimes \rho_{4}\right)$ we can still use the results of Part I and define the covariant four-potential operators of the electromagnetic field by $A_{i}(\phi)=\hbar q\left(H^{-1 / 2} \phi\right.$ $\left.\otimes \rho_{i}\right), i=1,2,3, \Phi(\phi)=-i \hbar p\left(H^{-1 / 2} \phi \otimes \rho_{4}\right)$ where $\phi \in \mathbb{R}_{2}\left(E_{3}\right)$ is in the domain of $k^{-1}$ and $k^{2}$ and $H=c k \otimes I$ is the Hamiltonian. $\hbar Q\left(H^{-1 / 2} \phi \otimes \rho\right)$ is a real-linear function of $\rho$, so these quantities transform correctly under the Lorentz group. The four-potential operators are observables because $\left(A_{i}(\phi)\right) \dagger$ $=\mathrm{g} \hbar q\left(H^{-1 / 2} \phi \otimes \rho_{i}\right) * \mathrm{~g}=\mathrm{g} \hbar q\left(H^{-1 / 2} \phi \otimes \rho_{i}\right) \mathrm{g}=\hbar q\left(I \otimes \mathfrak{g}^{\prime} H^{-1 / 2} \phi \otimes \rho_{i}\right) \quad$ (by $\quad(7)$ and Theorem 4) $=\hbar q\left(H^{-1 / 2} \phi \otimes \mathfrak{g}^{\prime} \rho_{i}\right)=A_{i}(\phi)$, and $(\Phi(\phi)) \dagger=\mathfrak{g}\left(-i \hbar p\left(H^{-1 / 2} \phi \otimes \rho_{4}\right)\right) * g$ $=g i \hbar p\left(H^{-1 / 2} \phi \otimes \rho_{4}\right) \mathfrak{g}=i \hbar p\left(I \otimes \mathfrak{g}^{\prime} H^{-1 / 2} \phi \otimes \rho_{4}\right)=i \hbar p\left(H^{-1 / 2} \phi \otimes \mathfrak{g}^{\prime} \rho_{4}\right)=i \hbar p\left(H^{-1 / 2} \phi\right.$ $\left.\otimes-\rho_{4}\right)=\Phi(\phi)$.

By Theorem 11, $\left(\left[A_{i}(\phi), A_{i}\left(\phi^{\prime}\right)\right]\right)^{\sim}=-\left(\left[\Phi(\phi), \Phi\left(\phi^{\prime}\right)\right]\right)^{\sim}=\left(\hbar^{2} / 2\right)\left(\left((1 / c k) \phi^{\prime}\right.\right.$, $\left.\phi)-\left(\phi,(1 / c k) \phi^{\prime}\right)\right) I$ and all of the cross-commutators are zero. But $\phi$ and $\phi^{\prime}$ will be distributions on $E_{3}$ with respect to which field-strength averages are computed, so they are both real-valued functions on $E_{3}$. Then $(1 / c k) \phi^{\prime}$ is also a real-valued function on $E_{3}$ and the inner product $\left((1 / c k) \phi^{\prime}, \phi\right)$ 
$=\left(\phi,(1 / c k) \phi^{\prime}\right)$ is real. Therefore, in all cases with which we will be concerned:

$$
\begin{aligned}
\left(\left[A_{i}(\phi), A_{j}\left(\phi^{\prime}\right)\right]\right)^{\sim}=\left(\left[A_{i}(\phi), \Phi\left(\phi^{\prime}\right)\right]\right)^{\sim}=\left(\left[\Phi(\phi), \Phi\left(\phi^{\prime}\right)\right]\right)^{\sim} & =0, \\
i, j & =1,2,3 .
\end{aligned}
$$

In order to derive Maxwell's equations for the case in which there are no charges present to interact with the field, let the energy be given by the operator $\Omega(H)$. Then the four-potential satisfies the Lorentz condition $\boldsymbol{\nabla} \cdot A-(1 / c)(\partial / \partial t) \Phi=0$ because by $(10)$ and Lemma 4 we have $(\boldsymbol{\nabla} \cdot \boldsymbol{A}$ $-(1 / c)(\partial / \partial t) \Phi)(\phi)=\left((\boldsymbol{\nabla} \cdot \boldsymbol{A})(\phi)-(i / c \hbar)([\Omega(H), \quad \Phi(\phi)])^{\sim}\right)^{\sim}=\left(A_{1}(-(\partial / \partial x) \phi)\right.$ $\left.+A_{2}(-(\partial / \partial y) \phi)+A_{3}(-(\partial / \partial z) \phi)\right)^{2}-(1 / c \hbar) \Phi(i c k \phi)=\left(Q\left(k_{x}\left(\left(-i /(c k)^{1 / 2} \phi\right) \otimes \rho_{1}\right)\right.\right.$ $+Q\left(k_{y}\left(\left(-i /(c k)^{1 / 2} \phi\right) \otimes \rho_{2}\right)+Q\left(k_{z}\left(\left(-i /(c k)^{1 / 2} \phi\right) \otimes \rho_{3}\right)+Q\left(k\left(\left(-i /(c k)^{1 / 2}\right) \phi\right)\right.\right.\right.$ $\left.\left.\otimes \rho_{4}\right)\right)^{\sim}$. But if $\psi=\phi_{1} \oplus \phi_{2} \oplus \phi_{3} \oplus \phi_{4} \in \mathfrak{P}$ and $\psi^{\prime}=k_{x} \phi^{\prime} \oplus k_{y} \phi^{\prime} \oplus k_{z} \phi^{\prime} \oplus k \phi^{\prime}$, then $\left(\psi, \psi \dagger^{\prime}\right)=\left(\phi_{1}, \quad k_{x} \phi^{\prime}\right)+\left(\phi_{2}, \quad k_{y} \phi^{\prime}\right)+\left(\phi_{3}, \quad k_{z} \phi^{\prime}\right)-\left(\phi_{4}, \quad k \phi^{\prime}\right)=\left(k_{x} \phi_{1}+k_{y} \phi_{2}\right.$ $\left.+k_{z} \phi_{3}-k \phi_{4}, \quad \phi^{\prime}\right)=\iiint\left(k_{x} \phi_{1}(\boldsymbol{k})+k_{y} \phi_{2}(\boldsymbol{k})+k_{z} \boldsymbol{\phi}_{3}(\boldsymbol{k})-k \boldsymbol{\phi}_{4}(\boldsymbol{k})\right) \boldsymbol{\phi}(\boldsymbol{k})^{*} d k_{x} d k_{y} d k_{z}$ $=\iiint 0 \phi^{\prime}(\boldsymbol{k})^{*} d k_{x} d k_{y} d k_{z}$ by (14) (applied to $\mathfrak{B}$ instead of $\mathfrak{B} \dagger$ ). So $\left(\psi, \psi^{\prime} \dagger\right)=0$. Therefore, if $f$ is any element of $\mathbb{S}$ representing a collection of photons (elements of $\mathfrak{B})$ and $f_{n}=\left(I^{(0)} \oplus I^{(1)} \oplus \cdots \oplus I^{(n)} \oplus \mathrm{O} \oplus \cdots\right) f$, we have $((\boldsymbol{\nabla} \cdot \boldsymbol{A}$ $-(1 / c)(\partial / \partial t) \Phi)(\phi) f, f \dagger)=\lim _{n}\left((\boldsymbol{\nabla} \cdot \boldsymbol{A}-(1 / c)(\partial / \partial t) \Phi)(\phi) f, f_{n} \dagger\right)=\lim _{n}\left(f,(1 / 2)^{1 / 2}\right.$ $\left.\left(\omega_{s}^{*}\left(\psi^{\prime}\right)+\omega_{s}\left(\psi^{\prime} \dagger\right)\right) f_{n} \dagger\right)\left(\right.$ letting $\left.\phi^{\prime}=-\left(i /(c k)^{1 / 2}\right) \phi \operatorname{in} \psi^{\prime}\right)=\lim _{n}(1 / 2)^{1 / 2}\left(\left(f, \omega_{s}^{*}\left(\psi^{\prime}\right) f_{n} \dagger\right)\right.$ $\left.+\left(\omega_{s}^{*}\left(\psi^{\prime} \dagger\right) f_{n}, f \dagger\right)\right)=\lim _{n} \quad(1 / 2)^{1 / 2}(0+0)=0$. The expectation values of $(\nabla \cdot A-(1 / c)(\partial / \partial t) \Phi)(\phi)$ are always zero when the particles are photons.

Further: $\left(\square^{2} A_{j}\right)(\phi)=\left(\nabla^{2} A_{j}(\phi)-(i / c \hbar)\left(\left[\Omega(H),(i / c \hbar)\left(\left[\Omega(H), A_{j}(\phi)\right]\right]\right)^{\sim}\right)^{\sim}\right)^{\sim}$ $=\left(A_{j}\left(\nabla^{2} \phi\right)-\left(1 / c^{2} \hbar^{2}\right) i\left(\left[\Omega(H), A_{j}(i c k \phi)\right]\right)^{\sim}\right)^{\sim}=\left(A_{j}\left(-\left(1 / \hbar^{2}\right) k^{2} \phi\right)-\left(1 / c^{2} \hbar^{2}\right) A_{j}\right.$ $\left.\left(-c^{2} k^{2} \phi\right)\right)^{\sim}=0$. Similarly $\left(\square^{2} \Phi\right)(\phi)=0$.

It is easily seen that if a photon is horizontally polarized (as previously defined), then the corresponding electric vector is horizontal also and the magnetic vector is vertical-both perpendicular to the direction of motion of the photon.

Planck's relation $E=h \nu$, where $E$ is the energy of a single photon and $\nu$ the frequency of the induced field, also follows directly.

Since $\Omega(A)$ and $q(\phi)$ do not in general commute, we are usually unable to determine, simultaneously, particle-like and wave-like quantities. This was to have been expected since the particle and wave concepts represent complementary (in the sense of Bohr) aspects of reality. For example, number-of-photons $N$ (a particle-like quantity) and frequency $\nu$ (a wave-like quantity) must obey an uncertainty relation $d N \cdot d \nu \geqq 1$. A spectroscope tries to determine the intensity (number-of-photons) of that light having a certain color (frequency). These two observables do not commute, so there is a natural broadening of all spectral lines. The same effect prohibits high fidelity radio transmission on a narrow broadcast band. The particle-like observables $\Omega(A)$ are reduced by each $\Re^{(n)}$, whereas the wavelike observables $E(\phi)$ and $H(\phi)$ are not-every reaction of the field upon a measuring instrument is accomplished by the annihilation and creation of photons. 


\section{BIBLIOGRAPHY}

1. N. Bohr and L. Rosenfeld, Zur Frage der Messbarkeit der Elektromagnetischen Feldgrössen, Det Kgl. Danske Videnskabernes Selskab. Matematisk-Fysiske Meddeleser (XII) vol. 8 (1933) pp. 3-65.

2. - Field and charge measurements in quantum electrodynamics, Physical Reviews vol. 78 (1950) pp. 794-798.

3. V. Fock, Konfigurationsraum und Zweite Quantelung, Zeitschrift für Physik vol. 75 (1932) pp. 622-647.

4. W. Heisenberg, The physical principles of the quantum theory, Chicago, 1930.

5. Y. Mimura and T. Hosokawa, Space, time, and laws of nature, Journal of Science of Hirosima University vol. 9 (1939) pp. 217-225.

6. F. J. Murray and J. von Neumann, On rings of operators, Ann. of Math. vol. 37 (1936) pp. 116-229.

7. J. von Neumann, Mathematische Grundlagen der Quantenmechanik, Berlin, 1932.

8. B. v. Sz. Nagy, Spektraldarstellung Linear Transformationen des Hilbertschen Raumes, Berlin, 1942.

9. B. L. van der Waerden, Die Gruppentheoretische Methode in der Quantenmechanik, Berlin, 1932.

10. G. Wentzel, Einfïhrung in die Quantentheorie der Wellenfelder, Vienna, 1943.

University of Chicago,

Chicago, Ill. 